\title{
A new formulation of the electric vehicle routing problem with time windows considering concave nonlinear charging function
}

\author{
Xiaorong Zuo ${ }^{\mathrm{a}}$, Yiyong Xiao ${ }^{\mathrm{b} *}$, Meng You ${ }^{\mathrm{b}}$, Ikou Kaku ${ }^{\mathrm{c}}$, Yuchun $\mathrm{Xu}^{\mathrm{d}}$ \\ ${ }^{\text {a }}$ China Aerospace Academy of Systems Science and Engineering, Beijing 100854, China \\ ${ }^{\mathrm{b}}$ School of Reliability and Systems Engineering, Beihang University, Beijing 100191, China \\ ${ }^{c}$ Department of Environmental Management, Tokyo City University, Yokohama, 224-8551, Japan \\ d School of Engineering \& Applied Science, Aston University, Birmingham, B4 7ET, United Kingdom
}

\begin{abstract}
The electric vehicle routing problem with time window (EVRPTW) is an extension of the traditional vehicle routing problem with time window (VRPTWs), where new features of electric vehicles are considered, such as limited battery capacities, lack of infrastructures, and long charging time. In this study, new technical formulations were presented for vehicle route selection and charging station visit, which reduces the formulation complexity without using duplicated dummy nodes or arcs. Besides, a new linearization method was developed that employs a set of secant lines to surrogate the concave nonlinear charging function with linear constraints. This method defines the charging time as a continuous variable and uses fewer variables than existing formulation in literature. A mixedinteger linear programming (MILP) model was developed for the EVRPTW and computational experiments on Solomon's VRPTW instances were conducted to verify the proposed model. The experimental results were compared with the results using traditional routing models, which showed that the proposed model can result in better EVs logistics schedules with higher charging time utilizations. One benchmark problem was updated with new best solution.
\end{abstract}

Keywords: Electric vehicle routing problem; mixed-integer linear programming; nonlinear charging function; continuous optimization

\section{Introduction}

The fast depletion of the fossil fuel resources has essentially caused the excessive emission of greenhouse gas (GHS) along with the pollution issues (Bahramara and Golpîra, 2018; Golpîra and Khan, 2019). Due to their environmental benefits, electric vehicles (EVs) have steadily gained a greater market share with a rapid growth rate, e.g., more than $50 \%$ annually in China in recent years. The new registration numbers of EVs (battery electric vehicle and plug-in hybrid electric vehicle) in China from 2010 to 2017 were 1.43, 5.07, 9.90, 15.34, 73.17, 207.38, 336.00 and 579.00 (in thousands) units, respectively (IEA, 2018). China's EV ownership was ranked first in the world in 2018 (Ma et al., 2019). The environmentally friendly characteristics of EVs, such as no greenhouse gas emissions, lower noise pollution, and high energy efficiency, can also help logistics companies to attain a green image, which is regarded as an important competitive factor by an increasing number of socially and environmentally aware customers (Desaulniers et al., 2016; Soleimani et al., 2018; Wang et al., 2018). Recent sustainability analysis and estimation methods of life cycle carbon emission by EVs can be found in Casals et al., (2016), Fernandez (2018), and Wu et al. (2018), and recent study of energy minimizing in scheduling problems (Fu et al., 2019).

${ }^{*}$ Corresponding author. Tel.: +86-010-82316003

E-mail addresses: zuoxiaorong710@163.com (Xiaorong Zuo); xiaoyiyong@buaa.edu.cn (Yiyong Xiao); youmdyx@buaa.edu.cn (Meng You); kakuikou@tcu.ac.jp (Ikou Kaku); y.xu16@Aston.ac.uk (Yuchun Xu) 
In addition to the environmental benefits, EVs are also cost effective in comparison with conventional vehicles powered by fossil fuel. The cost of electricity consumed by an EV is approximately one-tenth to one-seventh of the cost of fules consumed by a conventional vehicle when travelling the same distance (Xiao et al., 2019) ${ }^{1}$. Furthermore, if an $\mathrm{EV}$ is charged during the low electricity usage period (i.e., at midnight when the unit electricity price is only one-third of its normal price in some provinces of China), the cost can be further reduced by a considerable margin. This is an attractive characteristic in current situations that fuel costs account for a major part of the expenditure of logistics providers, e.g., approximately $40-60 \%$ of the transportation costs in general (Sahin et al., 2009). Thus, the EVs are strongly promoted in the logistics industry by Chinese government in order to reduce transportation costs, as well as to promote the Supply-Side Reform in China (Zhang et al., 2017). Currently, a large number of EVs are employed the fleets of logistics companies in China and they have become an important part of the road logistics system.

Although EVs have substantial economic and environmental benefits compared with fossil-fuel powered vehicles, a number of obstacles still hamper the large-scale adoption of EVs in logistics (Sweda et al., 2016). A major issue is the so-called "range anxiety" that describes the concern of drivers that the EV may run out of energy before reaching its destination or the next charging station (CS). This is because a logistics EV typically requires visits to a CS once or multiple times during its daily service because of the limited travel range. This issue is more important in the early stage of using EVs when the CS infrastructures are far less common than fossil fuel stations (Yu et al., 2011), and therefore leads to the using EVs in logistics is not an easy task and the routes needs to be deliberatively optimized.

Another problem is that charging an EV takes much longer than refilling a traditional gasoline vehicle (Strehler et al., 2017), because a drained EV battery will typically requires a couple of hours to get fully recharged. While the delivery time is extremely important for a logistics company, and such long recharging in the middle of a trip may considerably affect the delivery efficiency. Furthermore, the charging function of an EV battery is always nonlinear, which means that the charging current may drop rapidly as the state of charging (SOC) increases from a lower level to high. This nonlinear characteristic means it requires a varying charging time if starting from different levels of SOC despite charging for the same amount of electrical energy. Thus, when to visit a CS, where to find a CS, and how long a charging time should be arranged for an EV, are important practical factors of the nonlinear electric vehicle routing problem (EVRP) confronting modern logistic companies.

In order to address the new challenges associated with efficient delivery using EVs, such as arranging efficient visits to CSs along the travel route and determining the appropriate duration of charging, better planning and scheduling are required for EVs logistics. However, the existing EVRP models in literature for EVs logistics planning and scheduling do not consider many of those practically important factors, such as the nonlinear SOC-time charging function, the charging options of multiple visits of CSs with flexible charging time, and ensuring the battery SOC is above a safe level. To take into consideration of all these practical factors and bring forward truly optimized solutions for EVRP, a new comprehensive model was developed for the electric vehicle routing problem with time windows (EVRPTW) considering concave nonlinear charging function (CNCF) (EVRPTW-CNCF for short) in this study. New advantages of the proposed EVRPTW-CNCF model are on four folds as follows:

\footnotetext{
1 The amount of electricity (or gasoline) consumed by an EV (or a conventional car) for travelling $100 \mathrm{~km}$ at $60 \mathrm{~km} / \mathrm{h}$ is approximately $10-20 \mathrm{kWh}$ (or 10-20 liter), costing 5-10 (or 60-120) RMB according to the average electricity (or gasoline) price in China.
} 
(1) The charging time (when visiting a CS) is continuously optimized based on a given CNCF according to the overall requirements of the delivery tasks.

(2) EVs may be recharged by any amount of electricity from any level of battery SOC, while the charging time is used as a continuous variable.

(3) The CNCF is surrogate with a set of linear constraints with controllable accuracy, to bound the nonlinear relations between the charged electricity, charging time, and SOC level.

(4) An enhanced method is used to model the routing of EVs with unlimited visits to CSs and without using dummy CS copies

The above EVRPTW-CNCF was formulated as a mixed-integer linear programming (MILP) model and was verified of its efficiency and effectiveness through computational experiments. Some managerial insights and suggestions were provided to help logistics managers in improving the efficient utilization of their EVs.

The remainder of this paper is organized as follows. In Section 2, related literature review is provided. In Section 3, problem description is given for an overview of the EVRPTW-CNCF. In Section 4, the CNCF is modeled with an efficient linearization method. In Section 5, a new route formulation is provided with unlimited number of visiting times to CS and without using dummy CS copies. In Section 6, an MILP model of the EVRPTW-CNCF is developed with some propositions. In Section 7, computational experiments conducted are conducted based on Solomon's benchmark instances, and the solution results obtained under different parameter settings are compared and explained. Finally, the conclusion is given in Section 8.

\section{Related literature review}

\subsection{Vehicle Routing Problem}

The vehicle routing problem (VRP) is a well-known combinatorial optimization problem that involves arranging a set of optimal customer routes for a fleet of vehicles with the shortest total travel distance and lowest cost, and it was first introduced by Dantzig and Ramser (1959). Subsequently, the VRP and many of its variants have been extensively studied in the past 50 years by considering various practical factors, such as capacitated-VRP (CVRP), VRP with pickup and delivery, VRP with time windows (VRPTW), and time-dependent VRP (Laporte et al., 2000; Toth and Vigo, 2002; Golden et al., 2008; Lin et al., 2014; Madankumar and Rajendran, 2019; Letchford and SalazarGonzález, 2019). A recent review on VRP can be found in Gayialis et al. (2019). In previous decades, numerous new VRP models that consider the balance between monetary costs and environmental problems have attracted considerable interest from operations research professionals. These models include the energy-minimizing VRP proposed by Kara et al. (2007), emission-based time-dependent VRP by Figliozzi (2010) and Jabali et al. (2012), pollution routing problem by Bektas and Laporte (2011) and Demir et al. (2012), rechargeable VRP (RVRP) by Conrad and Figliozzi (2011), green VRP (GVRP) by Erdogan and Miller-Hooks (2012), fuel consumption rate considered CVRP by Xiao et al. (2012), low carbon routing problem by Zhang et al. (2015), green vehicle routing and scheduling by Xiao and Konak $(2015,2016,2017)$, and green home health care routing problem by FathollahiFard et al. (2018a, 2018b, 2019) and Erdem M., Koç (2019).

\subsection{Electric Vehicle Routing Problem}

The electric vehicle routing problem (EVRP) is among the latest extensions of VRPs, where it considers the use 
of EVs in the logistics distribution field. To the best of the authors' knowledge, Conrad and Figliozzi (2011) were the first to extend the traditional VRP to EVRP and they proposed the RVRP model, which assumes that the EVs in a fleet are allowed to recharge at certain customer locations. Erdoğan and Miller-Hooks (2012) proposed another version of EVRP called the GVRP, which involves using "alternative energy vehicles" (all types of environmentally friendly vehicles, including EVs) that need to visit energy-recharging stations during a trip. Schneider et al. (2014) proposed an EVRP model with time windows constraints and CSs, where they assumed that the battery has a constant consumption rate (a linear function of the distance travelled) and a constant battery recharging rate (the amount of charged electrical energy is proportional to the charging time). Felipe et al. (2014) modeled the EVRP with the possible selection of multiple charging technologies at CSs. In the charging mode, the charging time comprises two parts: a fixed charging time related to the technology selected and a variable charging time that is linearly dependent on the amount of energy to be recharged. Bruglieri et al. (2015) introduced the partial recharge policy into the EVRPTW so batteries must not always be fully recharged in order to guarantee higher flexibility along the route plan. Hiermann et al. (2016) modeled the EVRP with mixed types of EVs with different capacities, costs, and recharging rates (i.e., constants). Keskin and Çatay (2016) developed an adaptive large neighborhood search algorithm for the EVRPTW problem and used partial recharge strategies similar to those described by Bruglieri et al. (2015) and Hiermann et al. (2016). Schiffer and Walther (2017) modeled the electric location routing problem with time window considering partial recharging policy. To get fast refilled, battery-swapping based EVRP models were also developed in many applications (Yang and Sun, 2015; Hof et al., 2017, Jie et al., 2018). Xiao et al. (2019) modeled the EVRPTW considering the energy/electricity consumption rate.

\subsection{Modeling and solution approaches}

In a number of EVRP models, the EVs have swappable batteries and they are allowed to visit battery-exchange stations to swap their batteries within a very short battery-swapping time in order to avoid long charging times. Zheng et al. (2014) presented a framework for the optimal design of battery charging/swap stations in a distribution system. Adler and Mirchandani (2014) investigated an online routing system for EVs with multiple origin-destination pairs by considering battery swapping and reservation. Yang and Sun (2015) proposed a version of EVRP that simultaneously optimizes the routing plan and the selection of battery-exchange stations. Liao et al. (2016) studied the electric vehicle touring problem (EVTP) with battery swapping operations and developed a graph-theoretic algorithm for EVTP with fixed tours. Schiffer and Walther (2017) extended the EVRP model of Yang and Sun (2015) to include CS visits, time windows constraints, and partial recharging options. Zuo et al. (2017) neglected the influence of time on the EVRP model and assumed that an EV will receive a fully charged battery upon arriving at the CS.

In contrast to the RVRP model of Conrad and Figliozzi (2011) where the CSs are assumed to be located at customer sites, Erdoğan and Miller-Hooks (2012) first treated the CSs as a set of special customer nodes that EVs can either choose to visit (for charging) or not. They used multiple copies of CSs as dummy nodes to ensure that each CS can be visited multiple times by different EVs. However, Koç and Karaoglan (2016) emphasized that the duplicated dummy nodes in the Erdoğan-Miller-Hooks (2012) version of the GVRP increase the complexity of the problem. Thus, instead of using dummy CS copies, they introduced a new binary variable, $x_{i j k}$, to indicate whether an EV has visited the $k^{\text {th }} \mathrm{CS}$ in the arc from customers $i$ to $j$ (by $x_{i j k}=1$ ) or not (by $x_{i j k}=0$ ). This approach was also adopted by 
Leggieri and Haouari (2017). However, the introduction of the variable $x_{i j k}$ yields an optimization model with a high number of extra binary decision variable instances when the number of CSs is large. In this study, a more efficient way was presented to formulate the CS visit, with the same level of this formulation complexity as traditional VRPs (see Section 5.1).

Another challenge when modeling EVRP is determining the optimal charging time for an EV when visiting a CS, which requires treating the charging time as a continuous decision variable and allowing a partial charging policy. Thus, an EV charges only with the energy required to finish the deliveries. Schneider et al. (2014) extended the Erdoğan-Miller-Hooks version of the EVRP and introduced a linear charging function where the charged energy is assumed to be proportional to the charging time. Several other studies also used partial recharge strategies to model the EVRP. However, most of them assumed a linear charging function, including GVRP by Felipe et al. (2014), EVRPTW by Bruglieri et al. (2015), EVRPTW with nonlinear energy consumption rate mixed fleet by Goeke and Schneider (2015), pick-delivery EVRPTW by Lin et al. (2016), EVRPTW with partial recharge strategies by Keskin and Çatay (2016), and the electric location routing problem with time windows and partial recharging by Schiffer and Walther (2017). In all of the models mentioned above, the charging time is calculated with a constant charging rate, which does not conform to real situations. This is because most EVs have a CNCF and the charging time may increase sharply as more electrical energy is charged. Furthermore, even when charging with the same amount of electrical energy, the charging times can differ considerably when starting from different SOC levels. In this study, a new EVRPTW model is proposed to consider the CNCF based on a new and efficient linearization approach (see Section 4).

To the best of the authors' knowledge, Montoya et al. (2017) conducted the first investigation to formulate an EVRP model that considers a CNCF. However, four important aspects that need to be improved for practical reasons are not considered in this model. First, there is no consideration of time windows constraints, which are important from a practical viewpoint but they are difficult to model with a CNCF. Second, the cost function does not cover some important terms, such as the waiting time, electricity consumption cost, and driver's variable wage. Third, their model continues to duplicate CSs as dummy customer nodes, which increases the problem complexity and reduces the computational efficiency. Fourth, the linearization of the CNCF is considerably complex because four additional decision variables are introduced. In this study, a new method is proposed which uses only one additional decision variable.

Froger et al. (2017a, 2017b) modeled the EVRP considering nonlinear charging function with a multiple choice model that was firstly proposed by Croxton et al. (2003) and a reduced model that separated the total charging energy into multiple parts and projecting them onto the piecewise linear secant lines. Villegas et al (2018) developed a technician routing and scheduling problem with conventional and electric vehicles (TRSP-CEV) considering technicians with different skills that are necessary to take a lunch break during traveling on its own planning horizon. The TRSP-CEV problem modeled the SOC as a nonlinear function of the charging time by using a discrete nonlinear approximation that captured the nonlinear behavior of the charging. Heuristic algorithms were often used as solution approaches for EVRPs (or Green VRPs), such as variable neighborhood search combining tabu search (Schneider et al., 2014), adaptive large neighborhood search (Keskin and Çatay, 2016; Hiermann et al., 2016; Hof et al., 2017), Simulated Annealing (Xiao et al., 2015), graph-theoretic algorithm (Liao et al., 2016), Hybrid Genetic Algorithm 
(Xiao et al., 2017), and Keshtel Algorithm (Fathollahi-Fard and Hajiaghaei-Keshteli, 2018).

In the above reviewed works, most of the EVRP models take multiple copies of CSs as dummy nodes to transform the CS visits as the same as traditional customer visits, which have obvious drawbacks such as limited visit times and increased node number. While the improved formulation introduced by Koç and Karaoglan (2016) and Leggieri and Haouari (2017) has increased the formulation complexity by expanding the solution space by $k$ times (where $k$ is the CS number). Moreover, the nonlinear property of the charging function was not considered the in most existing EVRP models. The recent linearization approximation introduced in Montoya et al. (2017) is complex and difficult to follow as it introduced four additional variables, and their EVRP model is still using the dummy node setting. This study presents a new EVRP model that overcomes these drawbacks by not using the dummy nodes, without increasing the complexity, and efficient linearization of the nonlinear charging function. Detailed deductions of the formulation are addressed in Sections 4 and 5, and the new EVRP model is given in Section 6.

\section{Problem description of the EVRPTW-CNCF}

The EVRPTW-CNCF model is described on a directed graph $G=(N \cup C, A \cup B)$, where $N=\{0,1,2, \ldots, n\}$ is the set of nodes representing the customers and depot (denoted by 0 ), $C=\{n+1, n+2, \ldots, n+m\}$ is the set of nodes representing the CSs, $A=\{(i, j) \mid i, j \in N ; i \neq j\}$ is the set of customer/depot arcs, which are combination of the node pairs in $N$, and $B=\left\{(i, c) \mid i \in N \backslash\{0\}, c \in C_{i}\right\}$ is the set of arcs linking customers to CSs. Each $\operatorname{arc}$ in $A$, e.g., $(i, j)$ $\in A$, is associated with an average travel speed, $v_{i j}$, a travel time, $t_{i j}$, for traversing the arc, and an amount of electrical energy, $d_{i j}$, required for traversing the arc (measured by a standard distance unit (SDU) ${ }^{2}$ ). The parameters $v_{i j}, t_{i j}$, and $d_{i j}$ are assumed to be constants over each arc. A fleet of homogenous EVs can be dispatched to serve customers in $N$, which start from and return to the depot. Each customer, $i$, must be served once and it is associated with a demand $a_{i}$, a service duration time $z_{i}$, a time window $\left[h_{i}, e_{i}\right]$ in which the service must start, and a set $C_{i}, C_{i} \subset C$, of nearby CSs that an EV can visit to recharge its battery after leaving the customer $i$. Each CS is assumed to have an unlimited capacity and it can serve multiple EVs at the same time. All of the EVs at these stations are assumed to have an identical SOC-time curve which is assumed to be a CNCF. The EVs that leave the depot are fully charged, i.e., charged to the maximum battery capacity $L$, measured in SDUs. A safety threshold denoted by $\lambda$ is the energy level above which the energy in each EV should be maintained. An EV can detour to visit a $\mathrm{CS} c$ in $C_{i}$ for recharging after a customer, $i$, on its route is visited and it then returns to serve the next customer after recharging.

Thus, all EVs must: (1) determine the travel routes (by binary variable $x_{i j}$ ) to accomplish the required customer services, (2) incorporate proper detours of CS visits into the routes (by binary variable $y_{i}$ ) to allow the EVs being recharged, and (3) determine the optimal recharging time at a CS (by non-negative continuous variable $t^{\prime \prime}$ ) to maintain the battery SOC above a safe level through all routes. The objective of the EVRPTW-CNCF is to minimize a comprehensive cost function comprising the major expenditure of a logistics company from a practical perspective, including the related fixed costs per EV per driver such as the rental or hire rate, driver's daily rate, and vehicle depreciation or maintenance, as well as variable costs such as the electrical energy cost and driver's rate per hour/mile.

\footnotetext{
2 A metric representing the EV's battery capacity displayed on an odometer, which indicates the distance that the EV can travel under standard conditions, e.g., the NEDC.
} 
In the above EVRPTW-CNCF model, the road network/graph is assumed to be in a constant transportation environment, with known distance, statistics-based traffic flow, and pre-calculated travel time and electricity/energy consumption over each arc. This assumption is in line with many practical situations where traffic speed over different area regions/arcs may have different but constant patterns. While logistic companies may also regulate their drivers to drive at specified and fixed speeds according to road types. For an environment with time-varying traffic conditions, The EVRPTW-CNCF model can be combined with existing techniques to formulate the travel speed/time of EV over an arc as piece-wisely time-dependent patterns (Xiao et al., 2017) and continuously optimized speed variable (Xiao et al., 2019).

\section{Modeling the CNCF}

Montoya et al. (2017) first provided a version of formulation for the nonlinear charging function of EV, where they introduced four additional variables to represent a piecewise linear approximation with quite complicated formulations. In this section, a new linearization method is introduced for the CNCF. The proposed method involves using a set of secant lines to approximate the CNCF curve with linear constraints and with only one additional binary variable being introduced and without increasing the formulation complexity level.

\subsection{Bounding the charging time from a zero SOC}

The SOC of a battery is a concave function, $f(t)$, of the charging time, $t$, and it can be surrogated by a set (denoted as $R$ and indexed by $r$ ) of consecutive secant lines, as shown in Fig. 1, starting from SOC $=0 \%$ and ending at SOC $=100 \%$. Each secant line, $r \in R$, is represented by a pair comprising a slope, $K_{r}$, and intercept, $B_{r}$, formulated as $S O C=K_{r} \cdot t+B_{r}$. For a given SOC, $S_{i}$, the following linear constraints in Eq. (1) can be used to bound the minimum required charging time, $t_{i}$, for charging the battery from $\mathrm{SOC}=0 \%$ to $\mathrm{SOC}=S_{i}$ at charging station $i$.

$$
S_{i} \leq K_{r} \cdot t_{i}+B_{r} \quad \forall r \in R
$$

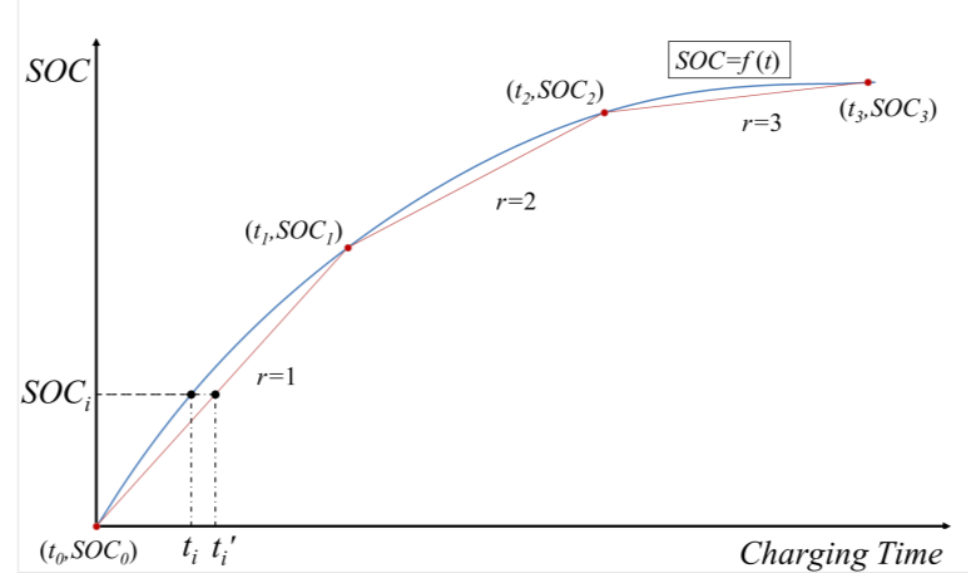

Fig. 1 Piecewise linearization of the SOC-time function (Source: TeslaFan(2015)).

Thus, Eq. (1) can be applied to an EVRP model where the objective is to minimize the charging time. It should be noted that Eq. (1) guarantees that the resulting charging time, $t_{i}{ }^{\prime}$, is always at the bottom-right side of the CNCF curve, so it will be greater than or equal to the accurate value $t_{i}$ (see Fig. 1). Thus, the final solution of the EVRP is guaranteed to be practically feasible from the perspective of time. It should be noted that the linearized approximation 
becomes more accurate as more secant lines are used.

\subsection{Bounding the charging time from a non-zero SOC}

However, the charging time needed to charge a given amount of electrical energy into the battery may vary considerably when starting from different levels of SOC. As shown in Fig. 2, to charge the battery with an amount, e.g., $\Delta S$, of electricity, different charging times, e.g., $\Delta t_{1}$ and $\Delta t_{2}$, are needed if starting from different SOCs, e.g., points $\mathrm{A}$ and $\mathrm{C}$. In the following, linear constraints are introduced to bound the minimum charging time (denoted by $\Delta t$ ) for charging the required amount of energy (denoted by $\Delta S$ ) into the battery starting from a non-zero SOC (denoted by $S^{\prime}$ ).

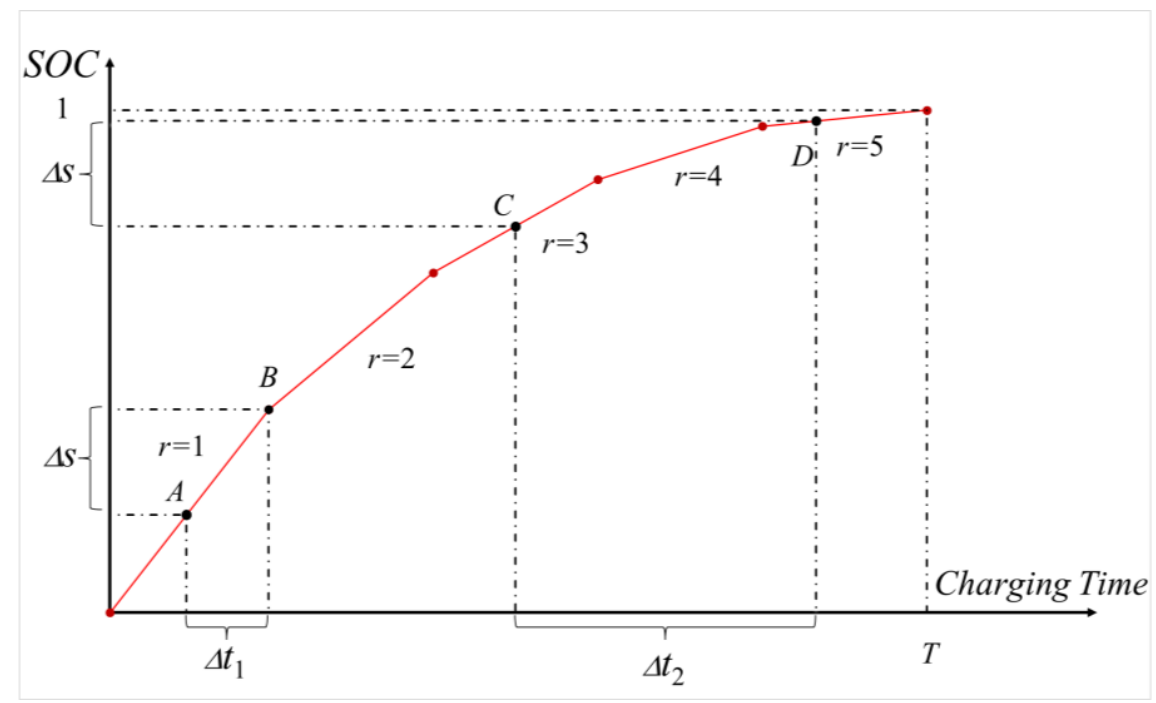

Fig. 2 Different charging time needed when starting from different SOCs.

Let $\left(t_{1}, s_{1}\right),\left(t_{2}, s_{2}\right), \ldots,\left(t_{k}, s_{k}\right)$, and $\left(t_{k+1}, s_{k+1}\right)$ be the breakpoints of the secant lines of the SOC curve, where $k$ represents the number of lines. Let $R$ represent the set of secant lines. Let $S^{\prime}$ be the current SOC of the battery, and $t^{\prime}$ be the charging time needed to charge the battery from SOC $=0$ to SOC $=S^{\prime}$. Let $\Delta S$ be the amount of electrical energy required to be charged into the battery, and $\Delta t$ be the charging time for charging a certain amount of electricity (i.e. $\Delta S$ ) from $\mathrm{SOC}=S^{\prime}$ to $\mathrm{SOC}=S^{\prime}+\Delta S$. Therefore, according to the charging curve in Fig.1, to charge the empty battery to $\mathrm{SOC}=S^{\prime}+\Delta S$, the corresponding total charging time is then $t^{\prime}+\Delta t$. Thus, Eq. (1) can be transformed into Eq. (2) as follows.

$$
S^{\prime}+\Delta S \leq K_{r} \cdot\left(t^{\prime}+\Delta t\right)+B_{r} \quad \forall r \in R
$$

It should be noted that Eq. (2) does not bound tightly the variable $\Delta t$ to $\Delta S$ because of the existences of variables $t^{\prime}$ and $S^{\prime}$. Next, a new binary variable, $\alpha_{r}$, representing whether $S^{\prime}$ satisfies $s_{r} \leq S^{\prime} \leq s_{r+1}$ (by $\alpha_{r}=1$ ) or not (by $\alpha_{r}=$ 0 ), is introduced to pick one secant line, $r \in R$, to surrogate the nonlinear relation between $t^{\prime}$ and $S^{\prime}$. Thus, the following Constraints (3) and (4) bound tightly variable $\alpha_{r}$ to $S^{\prime}$.

$$
\left\{\begin{array}{l}
S^{\prime} \geq s_{r}+\left(\alpha_{r}-1\right) \\
S^{\prime} \leq S_{r+1}+\left(1-\alpha_{r}\right)
\end{array} \quad \forall r \in R\right.
$$




$$
\sum_{r \in R} \alpha_{r}=1
$$

Constraint (3) ensures that if $S^{\prime}$ is between $s_{r}$ and $s_{r+1}$, then $\alpha_{r}=1$ must hold, whereas Constraint (4) ensures that $S^{\prime}$ belongs to only one of the value intervals $\left[s_{1}, s_{2}\right],\left[s_{2}, s_{3}\right], \ldots$, and $\left[s_{k}, s_{k+1}\right]$. On the contrary, if $\alpha_{r}=0$, Then the first constraint of Eq. (3) will transfer into $S^{\prime} \geq s_{r}-1$ which is always valid, because $S^{\prime}$ is a percentage denoting the battery level between $0 \%$ and $100 \%$. So, the right side of the inequality is always less than 0 . For the second constraint of Eq. (3), if $\alpha_{r}=0$, it will transfer into $S^{\prime} \leq s_{r}+1$ which is always valid, because the right side of the inequality must be greater than or equal to 1 . Thus, Eq. (3) will be make no restriction to $S^{\prime}$ if $S^{\prime} \leq s_{r}$ or $S^{\prime} \geq s_{r+1}$. Thus, by using constraints (3) and (4), a particular secant line, say $r$, where $r \in R$, is selected to represent the relation of variables $t^{\prime}$ and $S^{\prime}$. And then, the relation between $t^{\prime}$ and $S^{\prime}$ can be tightly bounded by the constraints in Eq. (5), as follows.

$$
\left\{\begin{array}{l}
S^{\prime} \geq K_{r} t^{\prime}+B_{r}-M\left(1-\alpha_{r}\right) \\
S^{\prime} \leq K_{r} t^{\prime}+B_{r}+M\left(1-\alpha_{r}\right)
\end{array} \quad \forall r \in R\right.
$$

It should be noted that $S^{\prime}$ is a percentage between 0 and 1 , so the big-M parameter $M$ can be set to 1 . Thus, by together using of Constraints (2)-(5), the minimum charging time (denoted by $\Delta t$ ) necessary to charge with the required amount of energy (denoted by $\Delta S$ ) in the battery starting from a non-zero SOC (denoted by $S^{\prime}$ ) is linearly bounded. Note that in the linear formulations given above, only one additional binary variable, i.e., $\alpha_{r}$, is added.

\section{Modeling the route with unlimited CS visits}

Erdogan and Miller-Hooks (2012) first modeled visits to alternative fueling stations (AFS) by copying the stations as multiple dummy nodes, where each is restricted to at most one visit by an EV. Thus, these AFSs can be visited as many times as the number of copied dummy nodes. This "copying" technique is in accordance with the traditional VRP frameworks and it has been included in many extended EVRP models (Felipe et al., 2014; Yang and Sun, 2015; Goeke and Schneider, 2015; Hiermann et al., 2016; Schiffer and Walther, 2017; Montoya et al., 2017). However, Koç and Karaoglan (2016) emphasized that the use of dummy nodes has disadvantages. First, it can increase the complexity of the problem to an extremely large size when the number of CSs is large. Second, the maximum number of dummy nodes for each CS cannot be known in advance, and thus it must be set as high as possible to avoid losing the potential benefit of multiple visits. However, this large number further increases the size of the set of dummy nodes and the problem's complexity.

Koç and Karaoglan (2016) abandoned the use of dummy nodes and introduced a new decision variable, $x_{i j c}$, to indicate whether a vehicle will visit CS $c$ as it traverses the arc from customer $i$ to customer $j$ (by $x_{i j c}=1$ ) or not (by $x_{i j c}=0$ ). Leggieri and Haouari (2017) improved the formulation of Koç and Karaoglan (2016) by considering the duration limit and they proposed a compact linearized formulation. However, the use of the variable $x_{i j c}$ also leads to a large number of decision variable instances for $x_{i j c}$ and the formulation has a complexity level of $\mathrm{O}\left(|N|^{2} \times|C|\right)$, where $N$ is the set of customers and $C$ is the set of CSs. The situation is worsened when the number of CSs is large. For example, there can be thousands of public CSs in some urban area. 
In the following, an efficient formulation of the EV visits to CSs is proposed, which maintains the number of decision variable instances at the same level of $\mathrm{O}\left(|N|^{2}\right)$ as the traditional VRP models.

\subsection{Efficient formulation for unlimited CS visits}

Let $N^{\prime}$ represent the set of customer nodes and $N=N^{\prime} \cup\{0\}$, where 0 denotes a unique depot. A set of nearby CSs is first predefined (denoted by $C_{i}$ and $C_{i} \subseteq C$ ) for each customer, $i \in N^{\prime}$, which includes only a limited number of CSs that are close to the customer of interest. A binary variable, $y_{i c}$, is then introduced to indicate whether a CS $c$ $\left(c \in C_{i}\right.$ ) will be visited after leaving customer $i$ (by $y_{i c}=1$ ) or not (by $y_{i c}=0$ ). Evidently, $\sum_{c \in C_{i}} y_{i c} \leq 1$. Assume that the maximum number of CSs near a customer is a constant, e.g., $z$. Consequently, the newly introduced variable, $y_{i c}$, leads to only $n \times z$ additional binary variable instances, where $n=\operatorname{card}\left(N^{\prime}\right)$. According to the proposed formulations, it is clear that the variable $y_{i c}$ is the only new independent decision variable introduced into the problem formulation. Therefore, the formulations for EVRPTW-CNCF have the same level of complexity as traditional VRPs, i.e., at a number of $\mathrm{O}\left(|N|^{2}\right)$ decision variable instances. It should be noted that the formulation complexity merely refers to the number of decision variables used, and not on the actual run time, which is still non-polynomial like all VRPs. The notation $O(\cdot)$ is used to indicate that the number of decision variables keeps in line with the same level of "." when the problem size increases. The linearization of the CNCF introduced in Section 4 has also an impact on the formulation complexity. However, it adds only a constant number, $|\mathrm{R}|$, of additional binary variables to the formulation and does not increase the formulation complexity. The level of variable number of different problem size is still determined by the major term $|N|^{2}$.

It is assumed that the electrical energy consumption when traversing a node arc $(i, j)$ is a constant, $d_{i j}$ (measured by SDU), where nodes $i$ and $j$ represent customers, depot, and CSs. A dependent variable denoted by $D_{i j}$ is used to represent the actual consumed energy (ACE) between nodes $i$ and $j$ if node $j$ is the next to be visited by an EV after node $i$. Thus, if an EV detours to visit a CS node, represented by $c$, after leaving customer $i$ and returns to serve customer $j$, then $D_{i j}$ is calculated by $D_{i j}=d_{i c}+d_{c j}$, where $d_{i c}$ and $d_{c j}$ represent the energy consumptions from customer $i$ to $\mathrm{CS} c$ and from CS $c$ to customer $j$, respectively; otherwise, $D_{i j}=d_{i j}$. An example is shown in Fig. 3 where two EVs are arranged to serve five customers. The EV with red lines and dash-dotted lines (represents detours to CSs) has visited two CSs with customer arcs $(0,5),(5,4),(4,3)$, and $(3,0)$, and its ACEs over the traversed arcs can be calculated by $D_{05}=d_{05}, D_{54}=d_{5 \mathrm{~B}}+d_{\mathrm{B} 4}, D_{43}=d_{4 \mathrm{~A}}+d_{\mathrm{A} 3}$, and $D_{30}=d_{30}$, respectively. The EV with the green line does not visit any CS, and thus the ACEs are equal to the constant energy consumed over the arcs. 


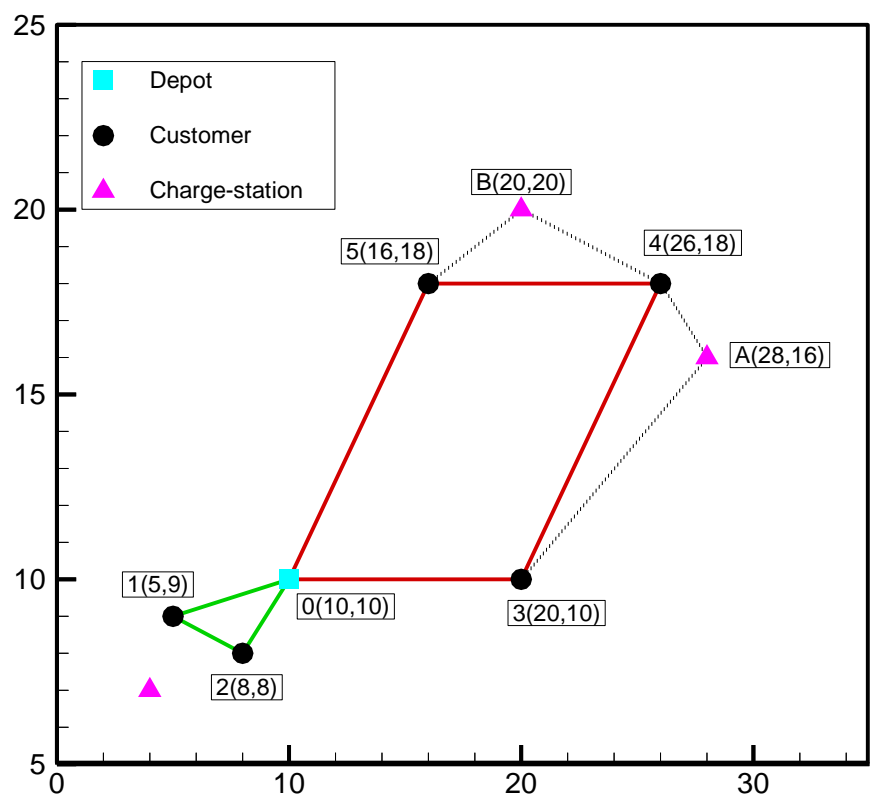

Fig. 3 Example comparing $d_{i j}$ and $D_{i j}$.

The binary variable, $x_{i j}$, is used to indicate whether customer $j$ is visited immediately after customer $i$ (by $x_{i j}=1$ ) or not (by $x_{i j}=0$ ), whereas the binary variable, $y_{i}^{\prime}$, i.e., $y_{i}^{\prime}=\sum_{c \in C_{i}} y_{i c}$, is used to indicate whether a CS is visited immediately after customer $i$ (by $y_{i}^{\prime}=1$ ) or not (by $y_{i}^{\prime}=0$ ). It is noteworthy that the variable $x_{i j}$ is not affected by whether a detour occurs to visit a CS from customer $i$, which is different from the settings used by Koç and Karaoglan (2016) and Leggieri and Haouari (2017). Thus, if an EV visits customer $i$ first, before detouring to visit CS $c$ and then visits customer $j$ from $c$, then $x_{i j}=1, y_{i c}=1$, and $y_{i}^{\prime}=1$. However, if EV visit customer $i$ and then customer $j$ without visiting any $\mathrm{CS}$ in between, then $x_{i j}=1$ and $y_{i}^{\prime}=0$.

Based on the definitions given above, Eq. (6) can be employed to tightly bound $D_{i j}$ with respect to the variables $x_{i j}$, $y_{i c}$, and $y_{i}^{\prime}$, as follows.

$$
D_{i j}=\left\{\begin{array}{ll}
x_{i j} d_{i j} & \text { if } y_{i}^{\prime}=0 \\
x_{i j} \sum_{c \in C_{i}}\left(d_{i c}+d_{c j}\right) y_{i c} & \text { if } y_{i}^{\prime}=1
\end{array} \quad \forall i \in N^{\prime}, j \in N\right.
$$

The expressions in Eq. (6) are nonlinear but they can be expressed with linear constraints using the big-M method. The linear constraints in Eqs. (16-1)-(16-5) are given in Section 6.

Next, assume that the travel time when traversing an $\operatorname{arc}(i, j)$ is a constant $t_{i j}$. Let $v_{i j}=d_{i j} / t_{i j}$, which represents the standard travel speed. Thus, another dependent variable, $T_{i j}$, is defined to represent the actual traverse time (ATT) for customer arc $(i, j)$. Let the variable $T_{i j}$ be calculated by $T_{i j}=D_{i j} / v_{i j}$, such that $T_{i j}=t_{i j}$ holds for the case where $D_{i j}=$ $d_{i j}$ and $T_{i j}=t_{i c}+t_{c j}$ holds for the case where $D_{i j}=d_{i c}+d_{c j}$.

Thus, the newly introduced variables $D_{i j}$ and $T_{i j}$, instead of the parameters $d_{i j}$ and $t_{i j}$, can be employed to model EVRP with unlimited CS visiting. It should be noted that the variables $D_{i j}, T_{i j}$, and $y_{i}^{\prime}$ are completely dependent on variables $x_{i j}$ and $y_{i c}$. Hence, they do not increase the combinational complexity of the solution space and the complexity of the formulation remains at the same level as the traditional VRPs. 


\subsection{Formulating energy recharging and consumption}

For each customer in $i \in N$, the variables $p_{i}, p_{i}^{\prime}$, and $p_{i}^{\prime \prime}$ represent the amount of remaining energy when arriving at customer $i$, the amount of remaining energy when arriving at a CS immediately after leaving customer $i$, and the amount of recharged energy at the CS immediately after leaving customer $i$, respectively. It should be noted that variable $p_{i}$ is meaningful only for customer nodes (excluding the depot and CSs), and the variables $p_{i}^{\prime}$ and $p_{i}^{\prime \prime}$ are meaningful only for those customer nodes after which a CS is directly visited, i.e., by $y_{i}^{\prime}=1$. It is assumed that the battery capacity of an EV is measured in SDUs. Thus, the values of variables $p_{i}, p_{i}^{\prime}$, and $p_{i}^{\prime \prime}$ all represent distances that the EV can travel under standard conditions. First, the variables $p_{i}$ and $p_{i}^{\prime}$ have the relationship shown in Eq. (7).

$$
p_{i}-p_{i}^{\prime}=\sum_{c \in C_{i}} y_{i c} d_{i c} \quad \forall i \in N^{\prime}
$$

Let $t_{i}^{\prime}$ be the charging time for the battery from zero to $p_{i}^{\prime}$. Then, $t_{i}^{\prime}$ and $p_{i}^{\prime}$ can be tightly bound to each other using the inequalities in Eq. (5), as illustrated in Section 4. Thus, according to Eq. (2), the charging time, $t_{i}^{\prime \prime}$, which is the charging time required to charge the battery from a lower SOC, i.e., $p_{i}^{\prime} / L$, to a higher SOC, i.e., $\left(p_{i}^{\prime}+\right.$ $\left.p_{i}^{\prime \prime}\right) / \mathrm{L}$, can be restricted from the lower bound side by the inequality in Eq. (8), as follows:

$$
\left(p_{i}^{\prime}+p_{i}^{\prime \prime}\right) / L \leq K_{r}\left(t_{i}^{\prime}+t_{i}^{\prime \prime}\right)+B_{r} \quad \forall i \in N^{\prime}, r \in R,
$$

where the parameter $L$ is the maximum battery capacity representing the maximum standard distance length a fully charged EV can travel. In order to guarantee that battery charging occurs only at customers where a CS will be visited immediately after and to consider the maximum battery capacity, the inequalities in Eq. (9) must be applied as follows.

$$
\left\{\begin{array}{l}
p_{i}^{\prime \prime} \leq L y_{i}^{\prime} \\
p_{i}^{\prime}+p_{i}^{\prime \prime} \leq L
\end{array} \quad \forall i \in N^{\prime}\right.
$$

Next, the energy consumption flow along the route is formulated. As mentioned above, it is assumed that the EVs are fully charged when leaving the depot, and thus they do not need to visit a CS. This assumption is reasonable from a practical viewpoint. Thus, Eq. (10) can be used to calculate the energy consumption flow along the route.

$$
\left\{\begin{array}{ll}
p_{i}=L-D_{0 i} & \text { if } x_{0 i}=1 \\
p_{i}=\sum_{j \in N^{\prime}} x_{i j}\left(p_{j}+D_{i j}-p_{i}^{\prime \prime}\right) & \text { if } x_{0 i}=x_{i 0}=0 \\
p_{i} \geq D_{i 0}+\lambda-p_{i}^{\prime \prime} & \text { if } x_{i 0}=1
\end{array} \quad \forall i, j \in N^{\prime}\right.
$$

The three equations in Eq. (10) can be used to calculate the amounts of remaining energy for the EVs that arrive at the first customer, the last customer, and customers excluding the first and last, respectively. The parameter $\lambda$ represents the safe threshold of the remaining energy when returning to the depot. To guarantee that the remaining energy remains at the same safety threshold when arriving at a CS, the inequality $p_{i}^{\prime} \geq \lambda y_{i}^{\prime}$ must be satisfied. It should be noted that Eq. (10) can be expressed in a linear manner using the big-M method (see the linear constraints in Eqs. (20-1)-(20-8) in Section 6). 


\section{A mixed-integer linear programming model}

Based on the methods proposed in Sections 5 and 6, the EVRPTW-CNCF is formulated as A MILP model and a number of theoretical propositions are provided regarding the solution obtained from the proposed model. The parameter notations and decision variables used to describe the EVRPTW problem are summarized as follows.

Parameter notations:

$N \quad$ set of customers (positive numbers) and depot (0)

$N^{\prime} \quad$ set of customers excluding the depot, $N^{\prime}=N \backslash\{0\}$.

$i, j \quad$ index of customers and depot, $i, j \in N$

C set of charging stations (CSs)

$C_{i} \quad$ subset of CSs that are close to customer/depot $i, C_{i} \subseteq C, i \in N$

A set of customer arcs that EVs can select to traverse, $i, j \in N ; i \neq j$

$B \quad$ set of arcs linking a customer/depot to a $\mathrm{CS}, \quad B=\left\{(i, c) \mid i \in N, c \in C_{i}\right\}$

$q \quad$ number of homogeneous EVs available in the fleet

$g_{v} \quad$ fixed cost rate per EV used

$C_{e} \quad$ maximum battery capacity of $\mathrm{EV}(\mathrm{kWh})$

$g_{e} \quad$ cost of electrical energy per unit $(\mathrm{kWh})$, including the electricity price and charging/parking fee

$g_{d} \quad$ cost of vehicle/driver per SDU of traveling distance

$g_{t} \quad$ cost of vehicle/driver per unit of delivery time

$O \quad$ maximum load capacity of an EV

$L \quad$ maximum travel range of an EV when fully charged (in SDUs)

$d_{i j} / d_{i c} d_{c j} \quad$ amount of electrical energy consumed over a node arc $(i, j) /(i, c) /(c, j)$ measured in SDUs, where $i$ and $j$ represent customers/depot and $c$ represent a CS

$t_{i j} \quad$ travel time required to traverse the $\operatorname{arc}(i, j)$

$v_{i j} \quad$ average speed over the $\operatorname{arc}(i, j), v_{i j}=d_{i j} / t_{i j}$

$R \quad$ set of consecutive secant lines that cut the nonlinear charging curve, $k=\operatorname{card}(R)$

$\left(s_{r}, \tau_{r}\right)$ the $r^{\text {th }}$ break-point in $R$, where $r=1,2, \ldots, k, k+1, s_{r}$ is the SOC (\%), and $\tau_{r}$ is the charging time

$K_{r}, B_{r} \quad$ slope and intercept of the $r^{\text {th }}$ secant line, respectively

$a_{i} \quad$ demand of customer $i$

$\left[h_{i}, e_{i}\right]$ time window to start serving customer $i$

$z_{i} \quad$ service time duration required to serve customer $i$

$M \quad$ a large number

$\lambda \quad$ safety threshold for the remaining energy of an EV measured in SDUs when arriving at a CS or the depot

Binary decision variables:

$x_{i j} \quad$ binary variable indicating whether $\operatorname{arc}(i, j)$ is traversed, $(i, j) \in A$

$y_{i c} \quad$ binary variable indicating whether $\operatorname{arc}(i, c)$ is traversed, $(i, c) \in B$

$y_{i}^{\prime} \quad$ binary variable indicating whether a CS is visited immediately after customer $i, \quad i \in N$ 
$\alpha_{i r} \quad$ binary variable indicating whether the SOC of an EV arriving at a CS immediately after customer $i$ is between $s_{r}$ and $s_{r+1}\left(\alpha_{i r}=1\right)$ or not $\left(\alpha_{i r}=0\right) ; i \in N^{\prime}$ and $r \in R$

Non-negative continuous decision variables:

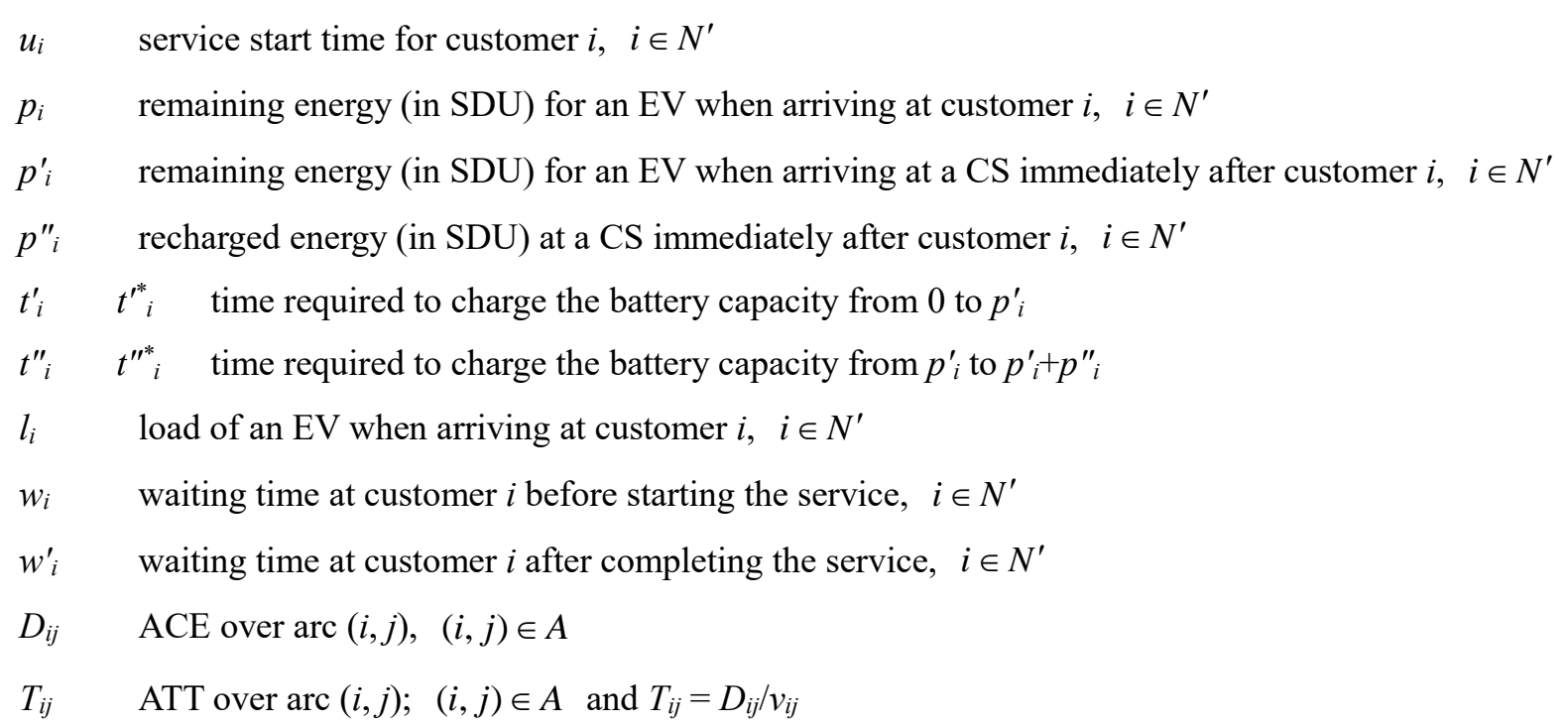

Problem EVRPTW-CNCF:

Min.

$$
\text { Total Cost }=g_{v} \sum_{(0, j) \in A} x_{0 j}+\left(g_{e} C_{e} / L\right) \sum_{(i, j) \in A} D_{i j}+g_{d} \sum_{(i, j) \in A} D_{i j}+g_{t}\left[\sum_{(i, j) \in A} T_{i j}+\sum_{i \in N^{\prime}}\left(w_{i}+z_{i}+w_{i}^{\prime}+t_{i}^{\prime \prime}\right)\right]
$$

Subject to:

$$
\begin{array}{ll}
\sum_{(j, i) \in A} x_{j i}=1 & \forall i \in N^{\prime} \\
\sum_{(i, j) \in A} x_{i j}=1 & \forall i \in N^{\prime} \\
\sum_{i \in N^{\prime}} x_{0 i} \leq q & \\
\begin{cases}y_{i}^{\prime}=\sum_{(i, c) \in B} y_{i c} & \forall i \in N \\
y_{0}^{\prime}=0 & \forall(i, j) \in A\end{cases} \\
\begin{cases}D_{i j} \geq d_{i j}-M\left(1-x_{i j}+y_{i}^{\prime}\right) & \forall(i, j) \in A \\
D_{i j} \leq d_{i j}+M\left(1-x_{i j}+y_{i}^{\prime}\right) & \forall(i, j) \in A,(i, c) \in B \\
D_{i j} \geq d_{i c} y_{i c}+d_{c j} y_{i c}-M\left(2-x_{i j}-y_{i c}\right) & \\
D_{i j} \leq d_{i c} y_{i c}+d_{c j} y_{i c}+M\left(2-x_{i j}-y_{i c}\right) & \forall(i, j) \in B\end{cases} \\
T_{i j}=D_{i j} / v_{i j} &
\end{array}
$$




$$
\begin{aligned}
& \begin{array}{ll}
u_{i} \geq T_{0 i}+w_{i}-M\left(1-x_{0 i}\right) & \forall(0, i) \in A \\
u_{i} \leq T_{0 i}+w_{i}+M\left(1-x_{0 i}\right) & \forall(0, i) \in A
\end{array} \\
& u_{i} \leq T_{0 i}+w_{i}+M\left(1-x_{0 i}\right) \quad \forall(0, i) \in A \\
& \left\{u_{j}-u_{i} \geq z_{i}+w_{i}+T_{i j}+t_{i}^{\prime \prime}+w_{j}^{\prime}-M\left(1-x_{i j}\right) \quad \forall(i, j) \in A, i>0, j>0\right. \\
& u_{j}-u_{i} \leq z_{i}+w_{i}+T_{i j}+t_{i}^{\prime \prime}+w_{j}^{\prime}+M\left(1-x_{i j}\right) \quad \forall(i, j) \in A, i>0, j>0 \\
& h_{i} \leq u_{i} \leq e_{i} \quad \forall i \in N^{\prime} \\
& l_{i} \geq a_{i} \quad \forall i \in N^{\prime} \\
& l_{i}-l_{j} \geq a_{i}-M\left(1-x_{i j}\right) \quad \forall(i, j) \in A, i>0, j>0 \\
& l_{i}-l_{j} \leq a_{i}+M\left(1-x_{i j}\right) \quad \forall(i, j) \in A, i>0, j>0 \\
& l_{i} \leq O \\
& \forall i \in N^{\prime} \\
& \forall(0, i) \in A \\
& \forall(0, i) \in A \\
& \forall(i, j) \in A, i>0, j>0 \\
& \forall(i, j) \in A, i>0, j>0 \\
& \forall(i, 0) \in A \\
& \forall i \in N^{\prime} \\
& \forall i \in N^{\prime} \\
& \forall i \in N^{\prime} \\
& \forall i \in N^{\prime}, r \in R \\
& \forall i \in N^{\prime} \\
& \forall i \in N^{\prime}, r \in R \\
& \forall i \in N^{\prime}, r \in R \\
& \forall i \in N^{\prime}, r \in R \\
& \forall i \in N^{\prime} \\
& \left\{\begin{array}{l}
x_{i j}, y_{i c}, y_{i}^{\prime}, \alpha_{i r} \in\{0,1\} ; u_{i} \geq 0 ; p_{i} \geq 0 ; p_{i}^{\prime} \geq 0 ; p_{i}^{\prime \prime} \geq 0 ; \\
t_{i}^{\prime} \geq 0 ; t_{i}^{\prime \prime} \geq 0 ; l_{i} \geq 0 ; w_{i} \geq 0 ; w_{i}^{\prime} \geq 0 ; D_{i j} \geq 0 ; T_{i j} \geq 0
\end{array} \quad \forall i, j, c, r\right.
\end{aligned}
$$

The formulae above are explained in the following. The linear objective function in Eq. (11) includes four components: (1) the fixed cost for vehicles, and drivers used and employed; (2) the variable cost of consumed electricity; (3) the variable cost related to the total travel distance; and (4) the variable cost related to the total delivery time. Constraints (12) and (13) ensure that each customer is visited and left only once. Constraint (14) restricts the number of EVs used in a solution so it does not exceed the available number. Constraint (15) indicates whether an EV selects a CS to visit after customer $i\left(y_{i}^{\prime}=1\right)$ or not $\left(y_{i}^{\prime}=0\right)$. In particular, $y_{0}^{\prime}$ is set to be zero indicating that all 
EVs are all fully charged before leaving the depot so they are not allowed to visit a CS right after. Constraints (161)-(16-4) are set of constraints for Eq. (6) to determine the ACEs over the arcs (see the illustration in Section 5.1). Constraint (17) determines the ATTs over the arcs. Constraints (18-1)-(18-5) restrict the time flow along the route and they ensure that each customer is served within their required time windows. Constraints (19-1)-(19-4) restrict the load flow along the route and they guarantee that the maximum load capacity of an EV is not exceeded. Constraints (20-1)-(20-8) restrict the battery energy flow along the route, based on which the remaining energy of an EV is calculated when arriving at the first customer (by Constraints (20-1) and (20-2)), when arriving at the last customer (by Constraints (20-5)), when arriving at the rest of the customers excluding the first and last (by Constraints (20-3) and (20-4)), and when arriving at a CS (by Constraints (20-6) and (20-7)). The constraints also ensure that the EVs have sufficient remaining energy above the safety threshold, $\lambda$, when returning to the depot (by Constraint (205)) and when arriving at a CS (By Constraint (20-8)). Constraints (21-1)-(20-6) use the linear approach introduced in Section 4 to bound the concave nonlinear relationship between the charging time and recharged energy. Constraints (21-1) and (21-2) determine one secant line, $r$ (by $\alpha_{i r}=1$ ), for each customer, $i$, which satisfies $s_{r} \leq p_{i}^{\prime} / L \leq s_{r+1}$. Constraints (21-3) and (21-4) compute the required charging time, $t^{\prime}$, according to the remaining energy, $p_{i}^{\prime}$, using the secant line determined by Constraints (21-1)-(21-2). Constraint (21-5) bounds the charging time from the lower level side with respect to the amount of charged energy. Constraint (21-6) ensures that the total energy of the battery (the remaining charge plus the added charge) does not exceed the maximum battery capacity. Constraint (22) defines the value domains of all the decision variables.

The following points should be noted. Constraints (16-2) and (16-3) apply only for $x_{i j}=1$ and $y_{i}^{\prime}=0$; Constraints (16-4) and (16-5) apply only for $x_{i j}=1$ and $y_{i}^{\prime}=1$. Constraints (18-1), (18-2), (20-1), and (20-2) apply only for $x_{0 i}$ $=1$. Constraints (18-3), (18-4), (19-2), (19-3), (20-3), and (20-4) apply only for $x_{i j}=1$. Constraint (20-5) applies only for $x_{i 0}=1$. Constraints (20-6), (20-7), (20-8), (21-5), and (21-6) apply only for $y_{i}^{\prime}=1$. Constraints (21-1), (21-3), and (21-4) apply only for $\alpha_{i r}=1$. The MILP model proposed above for the EVRPTW-CNCF formulated in Eqs. (11)-(22) can be solved optimally with MIP software, such as LINGO, LINDO, and CPLEX. Several propositions of the model were provided in Section Appendix.

\section{Computational experiments}

All of the computational experiments were performed using a Linux PC server with two $2.30 \mathrm{GHz}$ Intel Xeon (R) CPUs and 128-GB RAM. The mathematic programming language involved in the computational experiments is AMPL (A mathematical programming language, AMPL) that can describe optimization data, variables, objectives and constraints. The MIP solver CPLEX (version 12.6.0.1) was called by the AMPL language to directly solve all of the test instances.

\subsection{Test instances for EVRPTW}

Three sets of benchmark EVRPTW instances based on Solomon's VRPTW instances were generated, which are available at http://w.cba.neu.edu/ msolomon/problems.htm. These instances comprised R1-type, C1-type, and RC1type containing 101 nodes with specific coordinates, demands, time windows, and service times for each customer. The geographical data were randomly generated in problem sets R1, clustered in problem sets $\mathrm{C} 1$, and mixed with 
random and clustered structures in problem sets RC1. It was assumed that the energy consumed over an arc was equal to the arc distance (in SDUs) and the travel time was also equal to the arc distance number measured in minutes. The first 10 customers with a depot were chosen to form 10-customer instances for testing, i.e., $|N|=11$, as described in Sections 7.2, 7.3, and 7.4, and the Solomon instances with full customers, i.e., 100 customers with a depot $(|N|=101)$, were tested in Section 7.5. For each tested instance, four CSs scattered in four corners of the space were available to each customer. Examples of the locations of CSs for 20 customers are shown in Fig. 4 . The $x$-coordinates of the CSs were calculated as $\min \left\{x_{i}\right\}+0.25 \max \left\{\left|x_{i}-x_{j}\right|\right\}$ and $\min \left\{x_{i}\right\}+0.75 \max \left\{\left|x_{i}-x_{j}\right|\right\}$, and the $y$-coordinates were calculated as $\min \left\{y_{i}\right\}+0.25 \max \left\{\left|y_{i}-y_{j}\right|\right\}$ and $\min \left\{y_{i}\right\}+0.75 \max \left\{\left|y_{i}-y_{j}\right|\right\}$, where $i$ and $j$ represent customers. The coordinates of the CSs were all rounded to integers.

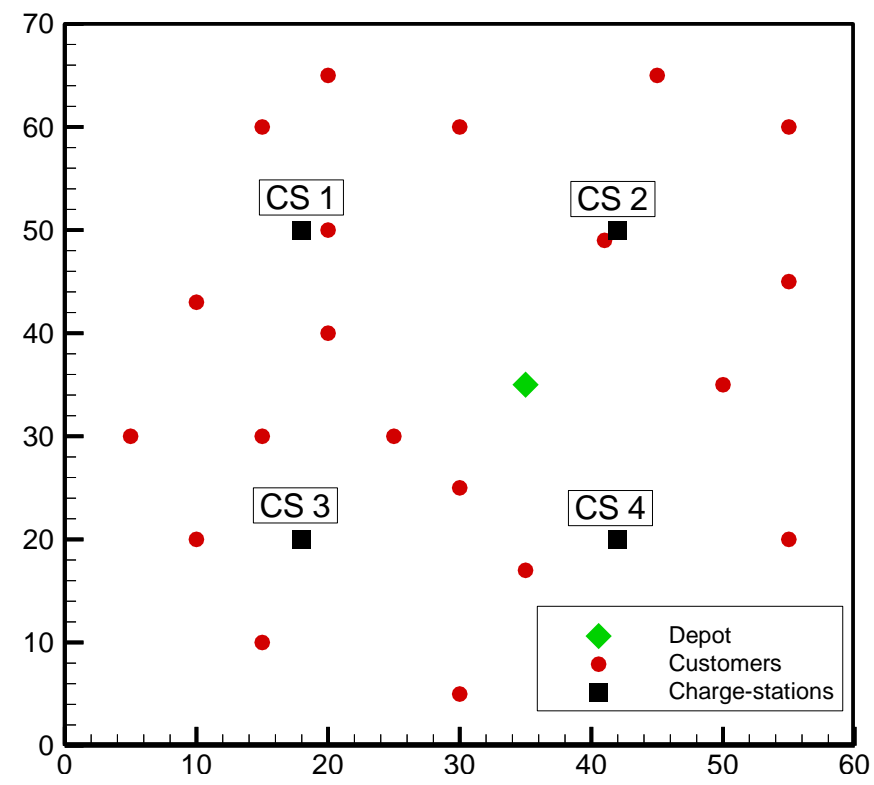

Fig. 4 Example of CS locations for 20 customers.

Assumed that the EVs had the CNCF provided by Teo TeslaFan available at Forums.tesla.2015 (https://forums.tesla.com/forum/forums/model-s-supercharging-times-compared-s60-s70d-s85-p85d-s85d). The CNFC indicates that the battery can be fully charged in 110 minutes. In the experiments, the numbers of secant lines used for linearizing the CNCF were set to $k=2,4,6$, and 8, as shown in Fig. 5. The state of charge for an EV was measured as the percentage charge, where the SOC of a fully charged battery was equal to $100 \%$. Moreover, in order to compare the obtained results with those solved for Solomon's instances as traditional VRPTW problems, the coefficients $g_{v}, g_{e}$, and $g_{t}$ of the objective function were set to be zero and $g_{d}=1$, so the obtained objective value would be the exact total distance traveled by the EVs. 


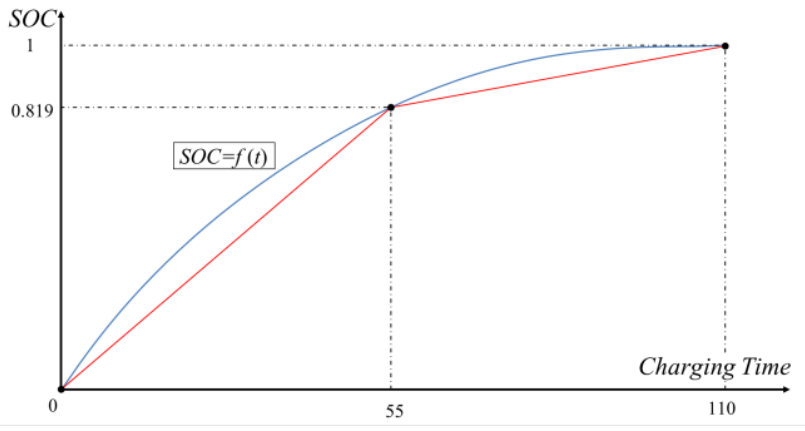

(A) $k=2$

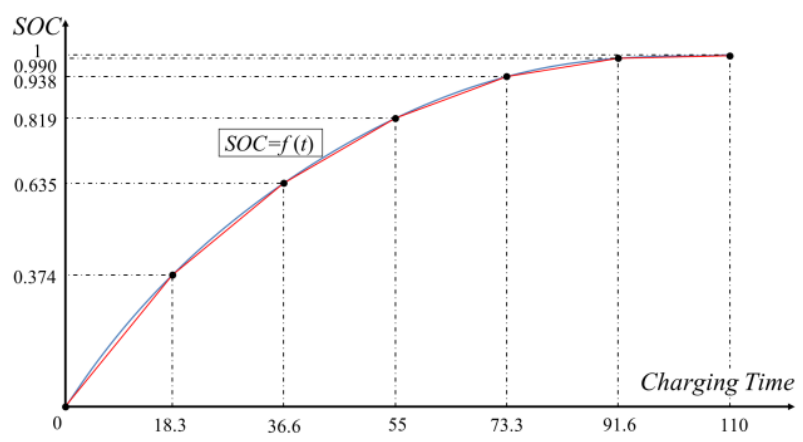

(C) $k=6$

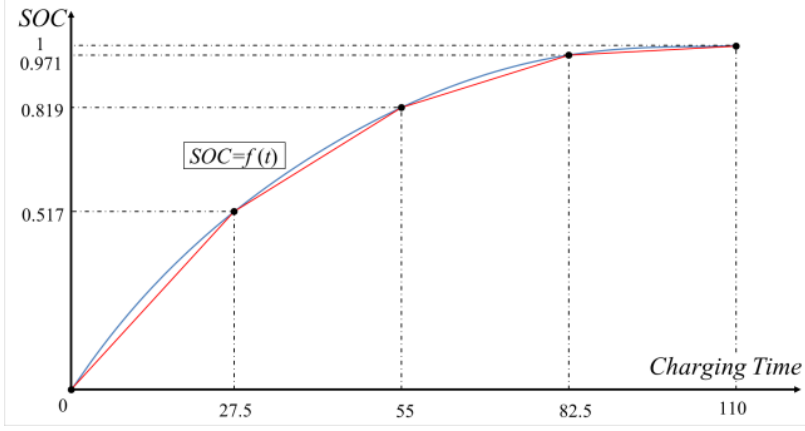

(B) $k=4$

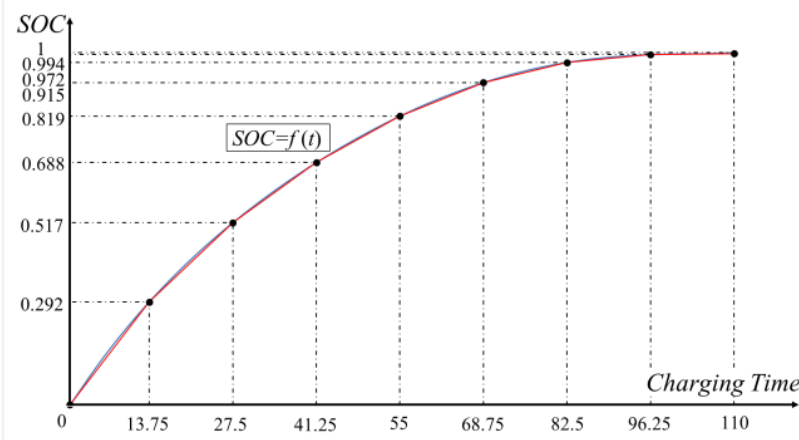

(D) $k=8$

Fig. 5 Piecewise linearization of the CNCF using $k=2,4,6$, and 8 secant lines.

\subsection{Effect of the travel range limit of EVS}

Solomon's instances were structured for fossil fuel-powered vehicles without travel range limits. However, EVs have limited battery capacities and they may need to visit a CS once or multiple times to complete the delivery. In the following experiments, the maximum range of an EV, denoted as $L$, were set to decrease from 150 to $20 \mathrm{~km}$, and three sets of Solomon's instances (i.e., R-type, C-type, and RC-type) were solved with the proposed MILP model by using the CPLEX solver. The other parameters were set to $k=2, O=50, m=10$, and $\lambda=5$. The states of the resulting solutions shown in Fig. 6 indicate whether a feasible solution was found. Moreover, the figure shows whether the EVs visited CSs under different values of the parameter $L$.

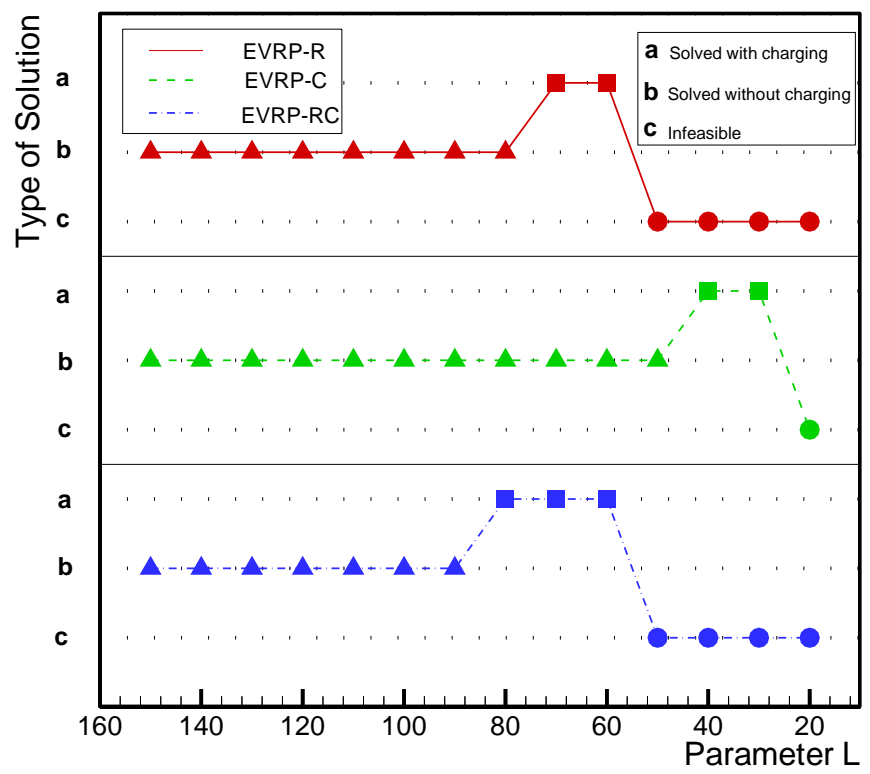

Fig. 6 States of solution with different values for the parameter $L$. 
Figure 6 shows that the states of the solutions varied as the parameter $L$ decreased. Initially, all of the problem types had optimal solutions even without visiting a CS when the parameter $L$ was $150 \mathrm{~km}$. However, as $L$ decreased, the EVs began to visit CSs in order to complete their deliveries, although a feasible route could not be found when $L$ decreased to extremely low values, e.g., $L=50 \mathrm{~km}$ for R-type and $L=20 \mathrm{~km}$ for C-type. Thus, the EVs had to visit CSs on their routes if $L$ was between 80 and $60 \mathrm{~km}$ for RC-type, between 40 and $30 \mathrm{~km}$ for C-type, or between 100 and $60 \mathrm{~km}$ for R-type.

The average objective values are plotted against the parameter $L$ for the test problems in Fig. 7. The dotted lines in Fig. 7 denote the average optimal objective value obtained by the conventional VRPTW without considering the travel range limit. The objective values tended to approach the objective value with VRPTW as the parameter $L$ increased, thereby indicating that an EV had to visit a CS when the parameter $L$ was sufficiently large.

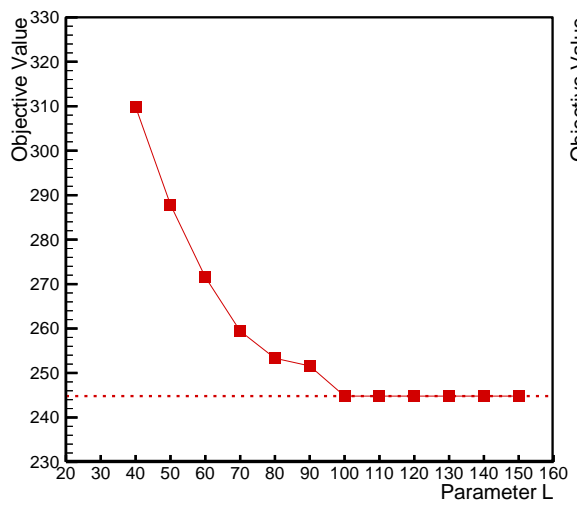

(A) EVRP_R-Type

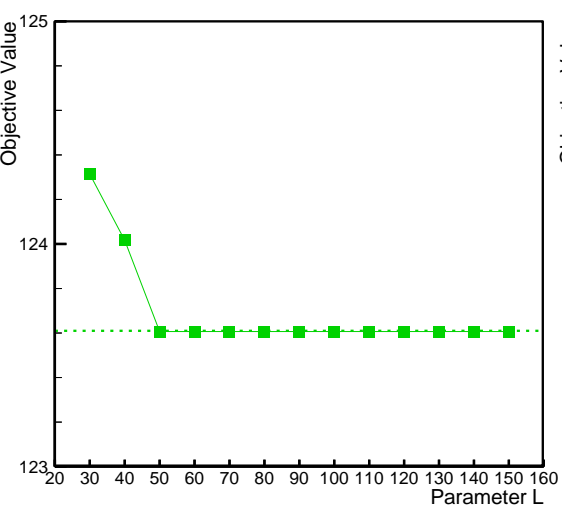

(B) EVRP_C-Type

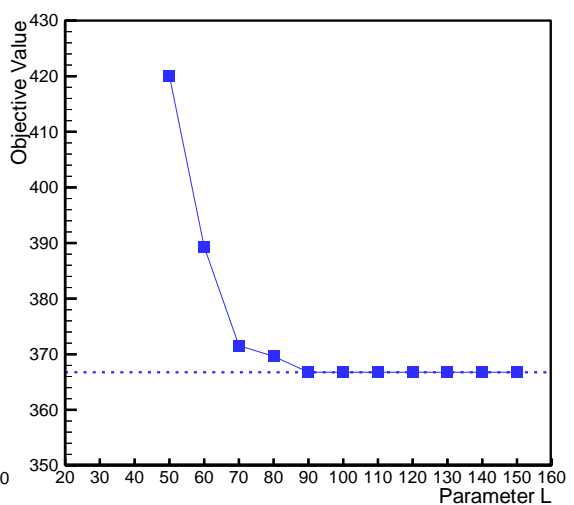

(C) EVRP_RC-Type

Fig. 7. State of solutions with respect to different values of the parameter $L$.

\subsection{Efficiency of the CNCF linearization}

Next, the performance of the MILP model was tested with different numbers of secant lines denoted as $k$ in the linearization of the CNCF. In general, the accuracy of the CNCF linearization was higher when more secant lines were used. However, a larger number of secant lines also consumed more CPU time. In these experiments, the Solomon's instances of C-type, R-type, and RC-type were solved with $k=2,4,6$, and 8 . The parameter $L$ was set to 30, 60, and 60 for C-type, R-type, and RC-type, respectively. The results are summarized in Table 1.

Table 1 Experimental results obtained with different $k$ values based on 10-customer instances (T-CT: total charging time, $|N|=11$ )

\begin{tabular}{|c|c|c|c|c|c|c|c|c|c|c|c|c|}
\hline \multirow{2}{*}{$\begin{array}{c}\text { Problem } \\
\text { No. }\end{array}$} & \multicolumn{3}{|c|}{$k=2$} & \multicolumn{3}{|c|}{$k=4$} & \multicolumn{3}{|c|}{$k=6$} & \multicolumn{3}{|c|}{$k=8$} \\
\hline & Obj. & T-CT & Time & Obj. & $\mathrm{T}-\mathrm{CT}$ & Time & Obj. & $\mathrm{T}-\mathrm{CT}$ & Time & Obj. & $\mathrm{T}-\mathrm{CT}$ & Time \\
\hline C101 & 125.25 & 189.52 & 2 & 125.25 & 194.32 & 3 & 125.25 & 192.61 & 3 & 125.25 & 193.37 & 3 \\
\hline C102 & 124.20 & 219.29 & 395 & 124.20 & 214.85 & 256 & 124.20 & 213.60 & 283 & 124.20 & 211.70 & 489 \\
\hline C103 & 124.20 & 219.29 & 250 & 124.20 & 214.85 & 268 & 124.20 & 213.60 & 267 & 124.20 & 211.70 & 336 \\
\hline C104 & 124.20 & 219.29 & 1380 & 124.20 & 209.83 & 1515 & 124.20 & 213.60 & 1591 & 124.20 & 211.70 & 1663 \\
\hline C105 & 124.20 & 222.42 & 1 & 124.20 & 209.83 & 3 & 124.20 & 214.55 & 3 & 124.20 & 211.70 & 4 \\
\hline C106 & 124.20 & 252.42 & 3 & 124.20 & 214.85 & 2 & 124.20 & 213.60 & 1 & 124.20 & 214.27 & 2 \\
\hline C107 & 124.20 & 219.29 & 2 & 124.20 & 209.84 & 3 & 124.20 & 213.60 & 3 & 124.20 & 211.70 & 3 \\
\hline C108 & 124.20 & 219.29 & 7 & 124.20 & 209.84 & 10 & 124.20 & 213.60 & 12 & 124.20 & 211.70 & 11 \\
\hline C109 & 124.20 & 219.29 & 26 & 124.20 & 209.84 & 37 & 124.20 & 213.60 & 40 & 124.20 & 211.70 & 44 \\
\hline AVG & 124.32 & 220.01 & 229.6 & 124.32 & 209.78 & 233 & 124.32 & 211.78 & 244.8 & 124.32 & 209.94 & 283.9 \\
\hline
\end{tabular}




\begin{tabular}{|c|c|c|c|c|c|c|c|c|c|c|c|c|}
\hline R101 & 325.32 & 42.77 & $<1$ & 325.32 & 64.37 & 1 & 325.32 & 64.5 & 1 & 325.32 & 64.13 & 1 \\
\hline R102 & 275.69 & 77.08 & 109 & 271.59 & 88.51 & 114 & 271.59 & 86.69 & 218 & 271.59 & 87.06 & 154 \\
\hline R103 & 275.69 & 77.08 & 107 & 271.59 & 88.51 & 94 & 271.59 & 86.69 & 115 & 271.59 & 87.06 & 142 \\
\hline R104 & 252.22 & 180.65 & 531 & 252.17 & 100.66 & 503 & 252.17 & 97.88 & 469 & 252.17 & 97.7 & 422 \\
\hline R105 & 321.57 & 86.94 & 3 & 321.6 & 98.46 & 3 & 321.57 & 85.26 & 6 & 321.57 & 85.97 & 5 \\
\hline R106 & 263.57 & 84.36 & 208 & 263.57 & 81.57 & 282 & 263.57 & 78.57 & 281 & 263.57 & 79.25 & 298 \\
\hline R107 & 263.57 & 84.36 & 150 & 263.57 & 81.57 & 228 & 263.57 & 78.57 & 234 & 263.57 & 79.25 & 282 \\
\hline R108 & 252.17 & 111.5 & 464 & 252.17 & 100.66 & 631 & 252.17 & 85.06 & 697 & 252.17 & 97.7 & 796 \\
\hline R109 & 263.87 & 63.83 & 15 & 263.87 & 54.86 & 16 & 263.87 & 51.98 & 22 & 263.87 & 53.04 & 14 \\
\hline R110 & 255.75 & 54.75 & 40 & 255.75 & 52.01 & 72 & 255.75 & 51.34 & 57 & 255.75 & 51.29 & 97 \\
\hline R111 & 255.63 & 69.11 & 198 & 255.63 & 73.07 & 180 & 255.63 & 92.77 & 232 & 255.63 & 87.07 & 277 \\
\hline R112 & 253.89 & 42.4 & 466 & 253.89 & 33.62 & 678 & 253.89 & 32.04 & 663 & 253.89 & 32.81 & 668 \\
\hline AVG & 271.58 & 81.24 & 190.9 & 270.89 & 76.49 & 233.5 & 270.89 & 74.28 & 249.6 & 270.89 & 75.19 & 263.0 \\
\hline RC101 & 396.11 & 135.46 & $<1$ & 396.11 & 115.1 & 1 & 396.11 & 115.85 & 1 & 396.11 & 116.05 & 1 \\
\hline $\mathrm{RC} 102$ & 388.15 & 126.55 & 9 & 388.15 & 108.04 & 11 & 388.15 & 109.35 & 11 & 388.15 & 109.8 & 12 \\
\hline $\mathrm{RC} 103$ & 388.15 & 126.55 & 8 & 388.15 & 108.04 & 14 & 388.15 & 109.35 & 10 & 388.15 & 109.8 & 15 \\
\hline $\mathrm{RC} 104$ & 388.15 & 126.55 & 12 & 388.15 & 108.04 & 15 & 388.15 & 109.35 & 28 & 388.15 & 109.8 & 17 \\
\hline $\mathrm{RC} 105$ & 388.15 & 126.55 & 7 & 388.15 & 108.04 & 8 & 388.15 & 109.35 & 8 & 388.15 & 109.8 & 11 \\
\hline $\mathrm{RC} 106$ & 389.86 & 128.47 & 5 & 389.86 & 109.56 & 8 & 389.86 & 110.75 & 6 & 389.86 & 111.14 & 7 \\
\hline $\mathrm{RC} 107$ & 388.97 & 127.47 & 11 & 388.97 & 108.77 & 11 & 388.97 & 110.02 & 10 & 388.97 & 110.44 & 15 \\
\hline $\mathrm{RC} 108$ & 387.25 & 125.55 & 14 & 387.25 & 107.25 & 14 & 387.25 & 108.62 & 17 & 387.25 & 109.1 & 16 \\
\hline AVG & 389.35 & 127.89 & 8.25 & 389.35 & 109.11 & 10.25 & 389.35 & 110.33 & 11.38 & 389.35 & 110.74 & 11.75 \\
\hline
\end{tabular}

Table 1 shows the total charging time and CPU time for each 10-customer instance, as well as the average total charging time and CPU time for each type of instance under different values of $k$. In addition, the differences in the average CPU time and total charging time obtained with $k=2$ are plotted against those obtained with other values of $k$ for all of the instance types in Fig. 8. The difference in the computational time increased as $k$ changed from 2 to 4 , whereas the growth rate was relatively flat from $k=4$ to $k=8$. As shown in Fig. 8(B), the charging time obtained with $k=2$ was considerably greater than those obtained with $k=4,6$, and 8 for all of the test problems, thereby indicating that using less secant lines could cause greater deviations from the actual values. These results indicate that eight secant lines was the best setting for use in the experiments because it approximated the CNCF curve and did not significantly increase the computational time. 


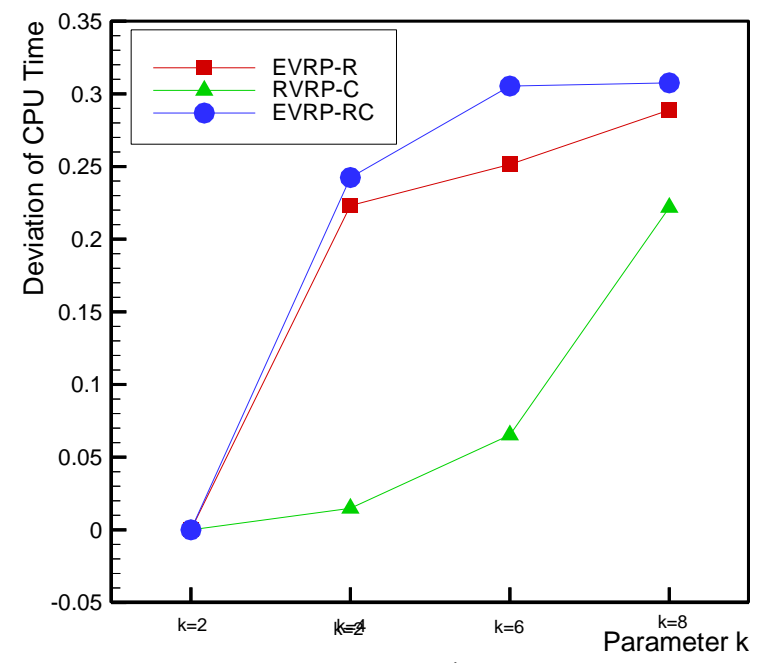

(A) CPU time

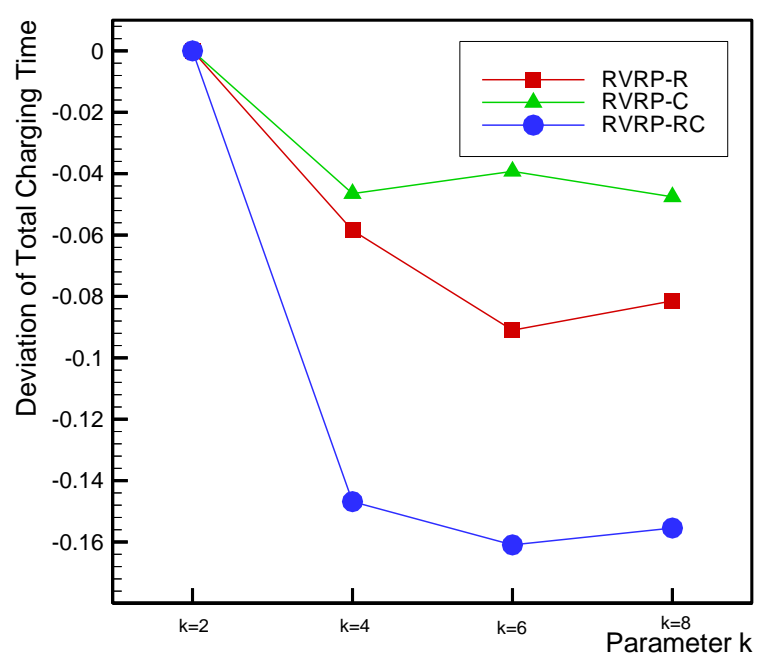

(B) Total charging time

Fig. 8 Trends in the CPU time and total charging time versus the parameter $k$.

In these experiments, except for EVRP_R102 and EVRP_R103, all of the test instances yielded the same objective values under different values of $k$. The objective values obtained with $k=2$ for EVRP_R102 and EVRP_R103 were greater than those obtained with $k=4,6$, and 8 . The detailed solutions for the R102 instance solved with $k=2$ and $k$ $=4$ are summarized in Tables 2 and 3, respectively, and the routing schemes are plotted in Fig. 9. The scheduled routes obtained with $k=2$ and $k=4$ were different, i.e., five EVs were used for $k=2$ but four for $k=4$. This difference occurred because the piecewise linearization with $k=4$ was more accurate than that with $k=2$. For example, the route comprising $0 \rightarrow 7 \rightarrow 8 \rightarrow 10 \rightarrow 0$ was feasible in the solution with $k=4$ but infeasible in the solution with $k=2$. As summarized in Table 4, the arrival times for customers 8 and 10 violated their time windows when only two secant lines were used to linearize $\mathrm{CNCF}$ at CS2 (i.e., $k=2$ ). This comparison confirmed that using more secant lines yielded better approximations in the $\mathrm{CNCF}$ linearization.

Table 2. Detailed solution for instance R102 (with $L=60 \mathrm{~km}, k=2, \mathrm{Obj},=275.70,|N|=11$ )

\begin{tabular}{cccccccccccccc}
\hline $\operatorname{arc}(i, j)$ & $D_{i j}$ & $a_{j}$ & $f_{j}$ & $p_{j}$ & $p^{\prime \prime}{ }_{i}$ & $t^{\prime}{ }_{i}$ & $t^{\prime \prime}{ }^{\prime}$ & $h_{j}$ & $u_{j}$ & $e_{j}$ & $z_{j}$ & $w_{j}$ & $w_{j}^{\prime}$ \\
\hline$(0,1)$ & 15.23 & 10 & 40 & 44.77 & -- & -- & -- & 0 & 15.23 & 204 & 10 & 0 & 15.72 \\
$(1, \mathrm{CS} 4)$ & 3.00 & - & 40 & 41.76 & 16.15 & 43.8 & 37.5 & -- & 43.95 & -- & -- & 0 & 0 \\
$(\mathrm{CS} 4,9)$ & 15.56 & 16 & 24 & 42.36 & -- & -- & -- & 97 & 97.1 & 107 & 10 & 0 & 0 \\
$(9,3)$ & 15.00 & 13 & 11 & 27.36 & -- & -- & -- & 0 & 122.1 & 197 & 10 & 0 & 0 \\
$(3,0)$ & 22.36 & 0 & 11 & 5 & -- & -- & -- & 0 & 144.46 & 230 & 0 & 0 & 0 \\
\hline$(0,2)$ & 18.00 & 7 & 43 & 42 & -- & -- & -- & 0 & 18 & 202 & 10 & 0 & 100.78 \\
$(2,4)$ & 20.22 & 19 & 24 & 21.78 & -- & -- & -- & 149 & 149 & 159 & 10 & 0 & 0 \\
$(4, \mathrm{CS} 3)$ & 13.60 & - & 24 & 8.18 & 8.23 & 7.25 & 7.30 & -- & 162.60 & -- & -- & 0 & 0 \\
$(\mathrm{CS} 3,0)$ & 11.40 & 0 & 24 & 5 & -- & -- & -- & 0 & 174 & 230 & 0 & 0 & 0 \\
\hline$(0,5)$ & 20.62 & 26 & 24 & 39.38 & -- & -- & -- & 0 & 20.62 & 199 & 10 & 0 & 0 \\
$(5,8)$ & 13.93 & 9 & 15 & 25.46 & -- & -- & -- & 95 & 95 & 105 & 10 & 50.46 & 0 \\
$(8,7)$ & 12.21 & 5 & 10 & 13.25 & -- & -- & -- & 0 & 117.21 & 198 & 10 & 0 & 0 \\
$(7, \mathrm{CS} 2)$ & 1.41 & - & 10 & 11.84 & 12.96 & 13.24 & 14.50 & -- & 118.62 & -- & -- & 0 & 0 \\
$(\mathrm{CS} 2,0)$ & 19.80 & 0 & 10 & 5 & -- & -- & -- & 0 & 138.42 & 230 & 0 & 0 & 0 \\
\hline$(0,6)$ & 11.18 & 3 & 47 & 48.82 & -- & -- & -- & 99 & 99 & 109 & 10 & 87.82 & 0 \\
$(6,0)$ & 11.18 & 0 & 47 & 37.64 & -- & -- & -- & 0 & 120.18 & 230 & 0 & 0 & 0 \\
\hline
\end{tabular}




\begin{tabular}{|c|c|c|c|c|c|c|c|c|c|c|c|c|c|}
\hline$(0,10)$ & 25.50 & 16 & 34 & 34.50 & -- & -- & -- & 124 & 124 & 134 & 10 & 98.50 & 0 \\
\hline$(10,0)$ & 25.50 & 0 & 34 & 9 & - & - & - & 0 & 159.50 & 230 & 0 & 0 & 0 \\
\hline \multicolumn{14}{|c|}{ Table 3 Detailed solution for instance R102 (with $L=60 \mathrm{~km}, k=4$, Obj. $=271.58,|N|=11$ ) } \\
\hline $\operatorname{arc}(i, j)$ & $\overline{D_{i j}}$ & $a_{j}$ & $\bar{f}$ & $p_{j}$ & $p^{\prime \prime}{ }_{i}$ & $\overline{t^{\prime} i}$ & $t^{\prime \prime}{ }_{i}$ & $\overline{h_{j}}$ & $\overline{u_{j}}$ & $\overline{e_{j}}$ & $\overline{z_{j}}$ & $\overline{w_{j}}$ & $\overline{w_{j}^{\prime}}$ \\
\hline$(0.1)$ & 15.23 & 10 & 40 & 44.77 & - & - & - & 0 & 15.23 & 204 & 10 & 0 & 15.72 \\
\hline (1.CS4) & 3 & - & 40 & 41.76 & 16.15 & 43.8 & 37.5 & - & 43.95 & - & - & 0 & 0 \\
\hline (CS4.9) & 15.56 & 16 & 24 & 42.36 & - & - & - & 97 & 97.1 & 107 & 10 & 0 & 0 \\
\hline (9.3) & 15 & 13 & 11 & 27.36 & - & - & - & 0 & 122.1 & 197 & 10 & 0 & 0 \\
\hline (3.0) & 22.36 & 0 & 11 & 5 & - & - & - & 0 & 144.46 & 230 & 0 & 0 & 0 \\
\hline$(0.2)$ & 18 & 7 & 43 & 42 & - & - & - & 0 & 18 & 202 & 10 & 0 & 100.78 \\
\hline (2.4) & 20.22 & 19 & 24 & 21.78 & - & - & - & 149 & 149 & 159 & 10 & 0 & 0 \\
\hline (4.CS3) & 13.60 & - & 24 & 8.18 & 8.23 & 7.25 & 7.30 & - & 162.60 & - & - & 0 & 0 \\
\hline (CS3.0) & 11.40 & 0 & 24 & 5 & - & - & - & 0 & 174 & 230 & 0 & 0 & 0 \\
\hline$(0.5)$ & 20.62 & 26 & 24 & 39.38 & - & - & - & 0 & 20.62 & 199 & 10 & 0 & 0 \\
\hline (5.6) & 10 & 3 & 21 & 29.38 & - & - & - & 99 & 99 & 109 & 10 & 58.38 & 0 \\
\hline$(6.0)$ & 11.18 & 0 & 21 & 18.20 & - & - & - & 0 & 120.18 & 230 & 0 & 0 & 0 \\
\hline$(0.7)$ & 21.21 & 5 & 45 & 38.79 & - & - & - & 0 & 21.21 & 198 & 10 & 0 & 0 \\
\hline (7.CS2) & 1.41 & - & 45 & 37.38 & 21.19 & 37.14 & 49.84 & - & 32.62 & - & - & 0 & 0 \\
\hline$(\mathrm{CS} 2.8)$ & 12.53 & 9 & 36 & 46.04 & - & - & - & 0 & 95 & 95 & 10 & 0 & 0 \\
\hline$(8.10)$ & 26.25 & 16 & 20 & 19.79 & - & - & - & 124 & 131.25 & 134 & 10 & 0 & 0 \\
\hline (10.CS2) & 14.21 & - & 20 & 5.58 & 19.22 & 4.95 & 17.05 & - & 155.46 & - & - & 0 & 0 \\
\hline$(\mathrm{CS} 2.0)$ & 19.80 & 0 & 20 & 5 & - & - & - & 0 & 175.26 & 230 & 0 & 0 & 0 \\
\hline
\end{tabular}

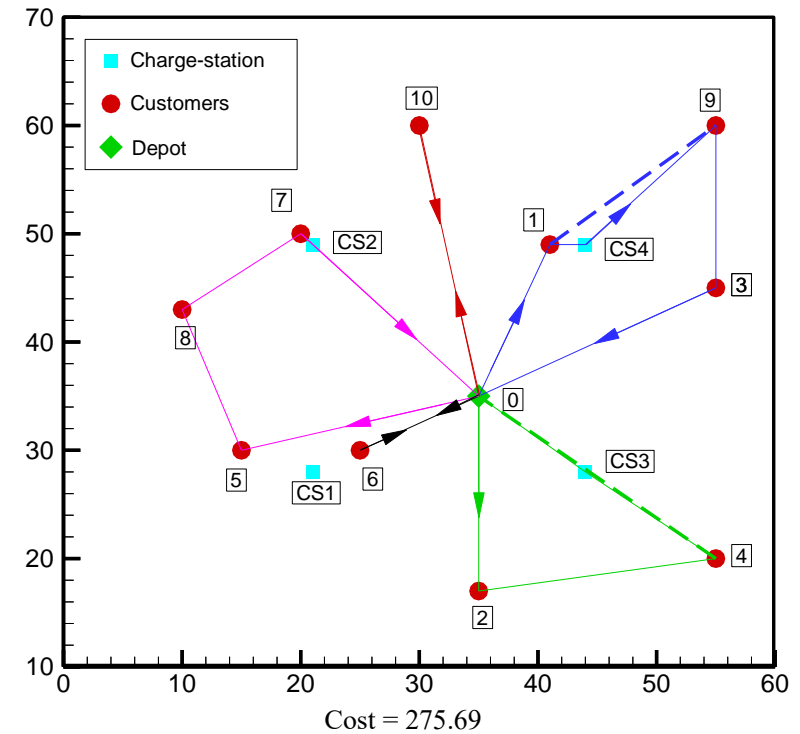

(A) Routing obtained with $k=2$

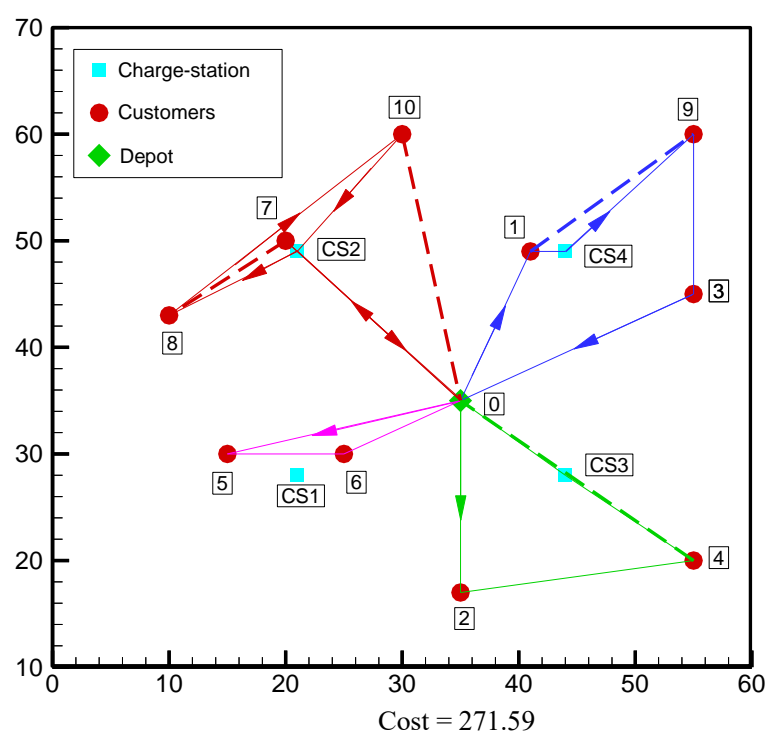

(B) Routing obtained with $k=4$

Fig. 9 Routing schemes obtained for the EVRP_R102 instance.

Table 4 Detailed routes obtained for customers 7, 8, and 10 for instance R102 (with $k=2$ )

\begin{tabular}{cccccccccccccc}
\hline $\operatorname{arc}(i, j)$ & $D_{i j}$ & $a_{j}$ & $f_{j}$ & $P_{j}$ & $P^{\prime \prime}{ }_{i}$ & $t^{\prime}{ }_{i}$ & $t^{\prime \prime}{ }_{i}$ & $h_{j}$ & $u_{j}$ & $e_{j}$ & $z_{j}$ & $w_{j}$ & $w_{j}^{\prime}$ \\
\hline$(0.7)$ & 21.21 & 5 & 45 & 38.79 & - & - & - & 0 & 21.21 & 198 & 10 & 0 & 0 \\
$(7 . \mathrm{CS} 2)$ & 1.41 & - & 45 & 37.38 & 20.61 & 41.81 & 58.66 & - & 32.62 & - & - & 0 & 0 \\
$(\mathrm{CS} 2.8)$ & 12.53 & 9 & 36 & 45.46 & - & - & - & 0 & $\mathbf{1 0 3 . 8 1}$ & 95 & 10 & 0 & 0
\end{tabular}




\begin{tabular}{cccccccccccccc}
$(8.10)$ & 26.25 & 16 & 20 & 19.21 & - & - & - & 124 & $\mathbf{1 4 0 . 0 6}$ & 134 & 10 & 0 & 0 \\
$(10 . \mathrm{CS} 2)$ & 14.21 & - & 20 & 5 & 19.80 & 5.59 & 22.15 & - & 164.27 & - & - & 0 & 0 \\
$(\mathrm{CS} 2.0)$ & 19.80 & 0 & 20 & 5 & - & - & - & 0 & 206.22 & 230 & 0 & 0 & 0 \\
\hline
\end{tabular}

To test the effect of the piecewise linearization applied on the charging function, a more convex shape of CNFC function (Type II as shown in Fig.10) was used to solve the eight RC-type instances with respect to $k=2$, 4, and 8 , respectively, and the obtained results were compared to those obtained under the Tesla's CNFC function (Type I). The type II CNFC was generated by applying the square root on the type I CNFC. All tested instances were solved to optimality by these two types of charging curves. The results are shown in Table 5, where column T.-CE indicates the total charged energy/electricity of all EVs (when visiting CSs) of the obtained solutions, column $T$.-CE* gives the exact value of total charged energy calculated by using the CNFC function with the same charging time, and column D.\% is the deviation of the linearized T.CE from the accurate T.CE*. It is observed that for both types I and II, using more secant lines to approximate the charging curve will result in lower deviation on total charged energy. The linearization accuracy of type $\mathrm{I}$ is around $1.2 \%$ on average for $k=8$, which is generally tolerable in practical applications. However, the solutions by type II curve have quite larger deviations than those by type I with respect to the same $k$. This is because type II has a larger curvature and hints that more secant lines (i.e., a large $k$ ) should be used in the linearization for a more convex CNFC function. Otherwise, it may cause a poor approximate performance.

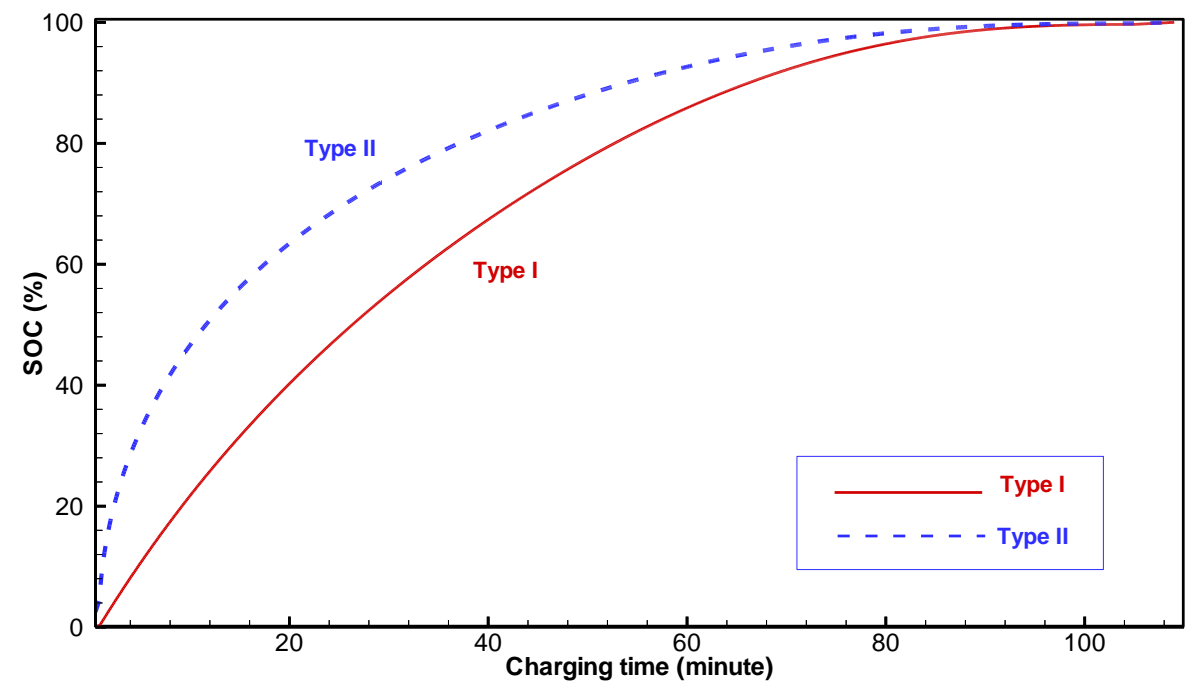

Fig. 10 Charging curves for two charging functions with different curvature.

Table 5 Experiments on the CNCF Linearization accuracy (with $k=2,4,8 ;|N|=11$ )

\begin{tabular}{|c|c|c|c|c|c|c|c|c|c|c|c|c|c|c|c|c|c|c|}
\hline \multirow{3}{*}{$\begin{array}{c}\text { Problem } \\
\text { No. }\end{array}$} & \multicolumn{9}{|c|}{ Type I } & \multicolumn{9}{|c|}{ Type II } \\
\hline & \multicolumn{3}{|c|}{$k=2$} & \multicolumn{3}{|c|}{$k=4$} & \multicolumn{3}{|c|}{$k=8$} & \multicolumn{3}{|c|}{$k=2$} & \multicolumn{3}{|c|}{$k=4$} & \multicolumn{3}{|c|}{$k=8$} \\
\hline & T-CE & T-CE* & D.\% & $\mathrm{T}-\mathrm{CE}$ & T-CE* & D.\% & T-CE & T-CE* & D.\% & $\mathrm{T}-\mathrm{CE}$ & T-CE* & D.\% & T-CE & T-CE* & D.\% & T-CE & T-CE* & D.\% \\
\hline RC101 & 121.1 & 137.8 & 13.8 & 121.1 & 122.2 & 0.9 & 121.1 & 122.4 & 1.1 & 121.1 & 170.8 & 41.1 & 121.1 & 137.4 & 13.4 & 121.1 & 128.1 & 5.7 \\
\hline $\mathrm{RC} 102$ & 113.1 & 127.8 & 13.0 & 113.1 & 114.9 & 1.6 & 113.1 & 114.4 & 1.1 & 113.1 & 159.0 & 40.5 & 113.1 & 127.2 & 12.4 & 113.1 & 121.5 & 7.4 \\
\hline $\mathrm{RC} 103$ & 113.1 & 127.8 & 13.0 & 113.1 & 114.9 & 1.6 & 113.1 & 114.4 & 1.1 & 113.1 & 159.0 & 40.5 & 113.1 & 127.2 & 12.4 & 113.1 & 121.5 & 7.4 \\
\hline $\mathrm{RC} 104$ & 113.1 & 127.8 & 13.0 & 113.1 & 114.9 & 1.6 & 113.1 & 114.4 & 1.1 & 113.1 & 159.0 & 40.5 & 113.1 & 127.2 & 12.4 & 113.1 & 121.5 & 7.4 \\
\hline $\mathrm{RC} 105$ & 113.1 & 127.8 & 13.0 & 113.1 & 114.9 & 1.6 & 113.1 & 114.4 & 1.1 & 113.1 & 159.0 & 40.5 & 113.1 & 127.2 & 12.4 & 113.1 & 121.5 & 7.4 \\
\hline $\mathrm{RC} 106$ & 114.9 & 130.1 & 13.2 & 114.9 & 116.8 & 1.7 & 114.9 & 116.2 & 1.2 & 114.9 & 161.5 & 40.6 & 114.9 & 129.6 & 12.8 & 114.9 & 122.7 & 6.9 \\
\hline $\mathrm{RC} 107$ & 114.0 & 129.0 & 13.2 & 114.0 & 115.8 & 1.6 & 114.0 & 115.5 & 1.3 & 114.0 & 160.3 & 40.6 & 114.0 & 129.3 & 13.5 & 114.0 & 121.8 & 6.9 \\
\hline $\mathrm{RC} 108$ & 112.3 & 126.8 & 13.0 & 112.3 & 114.0 & 1.6 & 112.3 & 113.7 & 1.3 & 112.3 & 157.7 & 40.5 & 112.3 & 126.9 & 13.1 & 112.3 & 120.5 & 7.4 \\
\hline
\end{tabular}




\subsection{EVRPTW-CNCF model with a fixed charging time}

In this experiment, a scenario comprising EVRPTW-NFC with a fixed charging time policy were considered, where EVs were only allowed to recharge at a CS for a given time duration, e.g., $30 \mathrm{~min}$. The solutions were compared with those obtained under a flexible charging time policy where EVs could be recharged as long time as necessary. This assumption is reasonable because it agrees with the facts found in a number of busy areas, where EVs may not be allowed (or be penalized) to occupy a CS for a long time.

A new parameter, denoted as $\rho$, was defined to indicate the fixed duration of charging time, and an additional constraint was added to the MILP model to restrict the charging time to being exactly equal to $\rho$, as follows.

$$
t_{i}^{\prime \prime}=\rho y_{i}^{\prime} \quad \forall i \in N^{\prime \prime}, \rho>0
$$

The constraint given above was only applied for $y^{\prime}=1$ and $\rho>0$, thereby ensuring that when a CS was selected to be visited after customer $i$, then the charging time, $t_{i}^{\prime \prime}$, was equal to $\rho$. All of the test instances were solved, where $\rho$ increased from 10 to 110 (stepped by 10 ), and the model was solved with the parameter $k=8$. The maximal travel range, $L$, was defined as equal to 30,60, and 80 for C-type, R-type, and RC-type, respectively. The solutions obtained with $\rho=20,30,40$, and 50 are summarized in Table 6, and compared with the solutions under the flexible charging time policy, which is indicated as "Cost by Fle.-C.T." The column "Dev.\%" represents the difference in an objective value compared with that obtained using the flexible charging time policy. For R-type, the average deviation increased from 0.75 to $2.11 \%$ as the parameter $\rho$ increased from 20 to 50 . However, the trends appeared to comprise a steady decline and steady flow for C-type and RC-type, respectively.

Table 6 Comparison of the results obtained for EVRP_RC-type with a flexible charging time policy (with $k=8,|N|=11$ )

\begin{tabular}{|c|c|c|c|c|c|c|c|c|c|}
\hline \multirow{3}{*}{$\begin{array}{c}\text { Problem } \\
\text { No. }\end{array}$} & \multirow{3}{*}{$\begin{array}{l}\text { Cost with } \\
\text { Fle.-C.T. }\end{array}$} & \multicolumn{8}{|c|}{ Cost with fixed charging time policy } \\
\hline & & \multicolumn{2}{|c|}{$\rho=20$} & \multicolumn{2}{|c|}{$\rho=30$} & \multicolumn{2}{|c|}{$\rho=40$} & \multicolumn{2}{|c|}{$\rho=50$} \\
\hline & & Cost & Dev.\% & Cost & Dev.\% & Cost & Dev.\% & Cost & Dev.\% \\
\hline EVRP_R101 & 325.32 & 325.32 & 0 & 331.95 & 2.04 & 331.95 & 2.04 & 331.95 & 2.04 \\
\hline EVRP_R102 & 271.59 & 275.69 & 1.51 & 275.69 & 1.51 & 275.69 & 1.51 & 271.59 & 0 \\
\hline EVRP_R103 & 271.59 & 275.69 & 1.51 & 275.69 & 1.51 & 275.69 & 1.51 & 271.59 & 0 \\
\hline EVRP_R104 & 252.17 & 253.89 & 0.68 & 253.89 & 0.68 & 253.89 & 0.68 & 253.89 & 0.68 \\
\hline EVRP_R105 & 321.57 & 323.05 & 0.46 & 321.6 & 0.01 & 321.57 & 0.00 & 331.95 & 3.23 \\
\hline EVRP_R106 & 263.57 & 264.52 & 0.36 & 271.78 & 3.11 & 271.78 & 3.11 & 271.59 & 3.04 \\
\hline EVRP_R107 & 263.57 & 264.52 & 0.36 & 271.78 & 3.11 & 271.78 & 3.11 & 271.59 & 3.04 \\
\hline EVRP_R108 & 252.17 & 253.89 & 0.68 & 253.89 & 0.68 & 252.17 & 0.00 & 253.89 & 0.68 \\
\hline EVRP_R109 & 263.87 & 264.27 & 0.15 & 269.95 & 2.30 & 273.28 & 3.57 & 277.93 & 5.33 \\
\hline EVRP_R110 & 255.75 & 257.49 & 0.68 & 258.86 & 1.22 & 255.75 & 0 & 267.85 & 4.73 \\
\hline EVRP_R111 & 255.63 & 262.16 & 2.55 & 255.63 & 0 & 255.63 & 0 & 262.16 & 2.55 \\
\hline EVRP_R112 & 253.89 & 253.89 & 0 & 253.89 & 0 & 253.89 & 0 & 253.89 & 0 \\
\hline AVG & 270.89 & 272.87 & 0.75 & 274.55 & 1.35 & 274.42 & 1.2 & 276.66 & 2.11 \\
\hline EVRP_C101 & 125.25 & 131.63 & 5.09 & 126.54 & 1.03 & 125.78 & 0.42 & 125.78 & 0.42 \\
\hline EVRP_C102 & 124.20 & 125.70 & 1.21 & 124.94 & 0.60 & 124.94 & 0.60 & 124.20 & 0 \\
\hline
\end{tabular}




\begin{tabular}{cccccccccc} 
EVRP_C103 & 124.20 & 125.70 & 1.21 & 124.94 & 0.60 & 124.94 & 0.60 & 124.20 & 0 \\
EVRP_C104 & 124.20 & 125.70 & 1.21 & 124.94 & 0.60 & 124.94 & 0.60 & 124.20 & 0 \\
EVRP_C105 & 124.20 & 131.06 & 5.52 & 125.98 & 1.43 & 125.78 & 1.27 & 125.25 & 0.85 \\
EVRP_C106 & 124.20 & 131.63 & 5.98 & 126.54 & 1.88 & 125.78 & 1.27 & 125.25 & 0.85 \\
EVRP_C107 & 124.20 & 131.06 & 5.52 & 125.98 & 1.43 & 125.78 & 1.27 & 125.25 & 0.85 \\
EVRP_C108 & 124.20 & 131.06 & 5.52 & 125.98 & 1.43 & 125.78 & 1.27 & 125.25 & 0.85 \\
EVRP_C109 & 124.20 & 130.87 & 5.37 & 125.98 & 1.43 & 125.78 & 1.27 & 125.25 & 0.85 \\
AVG & 124.32 & 129.38 & 4.07 & 125.75 & 1.16 & 125.50 & 0.95 & 124.96 & 0.52 \\
\hline EVRP_RC101 & 371.37 & 372.23 & 0.23 & 371.37 & 0 & 371.37 & 0 & 371.37 & 0 \\
EVRP_RC102 & 368.57 & 368.57 & 0 & 368.57 & 0 & 368.57 & 0 & 368.57 & 0 \\
EVRP_RC103 & 368.57 & 368.57 & 0 & 368.57 & 0 & 368.57 & 0 & 368.57 & 0 \\
EVRP_RC104 & 368.57 & 368.57 & 0 & 368.57 & 0 & 368.57 & 0 & 368.57 & 0 \\
EVRP_RC105 & 368.57 & 368.57 & 0 & 368.57 & 0 & 368.57 & 0 & 368.57 & 0 \\
EVRP_RC106 & 371.37 & 371.37 & 0 & 371.37 & 0 & 371.37 & 0 & 371.37 & 0 \\
EVRP_RC107 & 371.37 & 371.37 & 0 & 371.37 & 0 & 371.37 & 0 & 371.37 & 0 \\
EVRP_RC108 & 368.57 & 368.57 & 0 & 368.57 & 0 & 368.57 & 0 & 368.57 & 0 \\
AVG & 369.62 & 369.73 & 0.03 & 369.62 & 0 & 369.62 & 0 & 369.62 & 0 \\
\hline
\end{tabular}

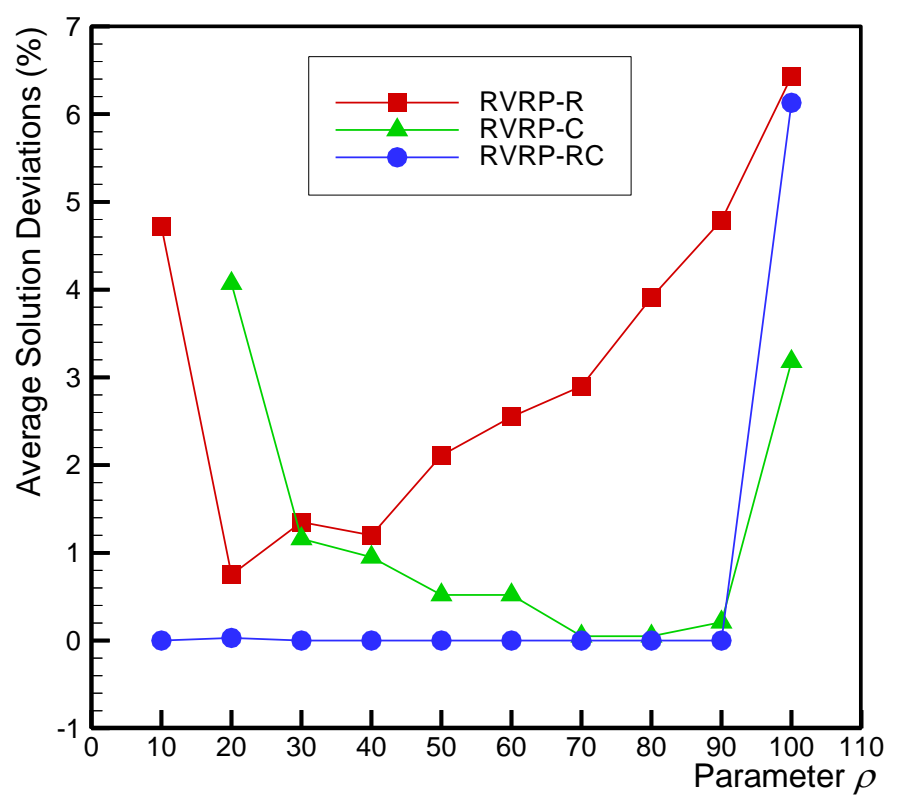

Fig. 11 Trends in the differences in the solutions with respect to different $\rho$ values.

Figure 11 shows the trends in the average deviations with respect to different values of the parameter $\rho$ and different problem types. Clearly, the trends determined for R-type and C-type decreased initially, before increasing as the parameter $\rho$ increased from 10 to 110 . However, RC-type exhibited a stable trend until $\rho$ reached 100 . Moreover, the maximum average deviation was reached when $\rho$ increased to 100 . These results demonstrate that the appropriate values for $\rho$ were 20,70, and 10 for EVRP_R, EVRP_C, and EVRP_RC, respectively. This comparative experiment may help logistics managers to use a fixed charging time policy to simplify logistics operations while also maintaining the high utilization of EVs.

\subsection{Efficiency of the EVRPTW-CNCF model}


Next, full-sized Solomon VRPTW instances (with 100 customers) were used to test the efficiency of the proposed EVRPTW-CNCF model in computational experiments. The maximum range limit for a fully charged EV was set to a large number, e.g., $L=2000$, so the EVs did not need to visit a CS and the total distance traveled for the solution was comparable to the solutions obtained with traditional heuristics for VRPTW models. The MIP solver CPLEX was applied to solve all 29 instances with a two hour time limit. After that, the maximum range limit parameter $L$ was set to a regular range, e.g., $L=150$, such that the EVs might need to visit CSs in order to complete their tours. Table 7 summarizes the computational results, including the deviation from the best solution obtained by the heuristics, the CPU time used, and relative mixed-integer optimality gap (Gap\%). The best known solutions solved by different authors identified by previous heuristics (available at http://w.cba.neu.edu/ msolomon/heuristi.htm) are also listed for comparison. According to Table 7, the EVRPTW-CNCF model could obtain feasible solutions for 25 and 19 (out of 29) of the tested instances for parameter $L=2000$ and $L=150$, respectively. For $L=2000$, the obtained solutions for instances of $\mathrm{C}$ type were all identical to the previous best solutions by heuristics, although the values for Gap \% were still large. While the obtained solutions of $\mathrm{R}$ and $\mathrm{RC}$ types were worse than the previous best solutions, and feasible solutions were even not found for the R110, R112, RC107, and RC108 instances. The solutions obtained by $L=150$ might contain detours to CS so they were not comparable to existing heuristic solutions. The experiments also indicated that it looked more difficult to solve the instances under $L=150$, as fewer feasible solutions were found in the time limit. This is because a smaller $L$ had tightened the searching space, and the solver needed more CPU time to initiate a feasible solution before starting the Branch \& Bound search process. However, a new best solution, i.e., 1642.88, surprisingly was found for R101 in the experiment. The detailed routes of the new best solution were provided in Fig.A1 in Appendix. The above experiments showed that the EVRPTW-CNCF model was applicable to solve large-sized instances. Moreover, it should be notable that instead of using full optimization at one time, some fix-and-optimize heuristics such as introduced in Xiao et al., $(2017,2019)$ could be employed to improve the computational efficiency by using the MILP-based partial optimizations.

\begin{tabular}{|c|c|c|c|c|c|c|c|c|c|c|}
\hline \multirow{2}{*}{$\begin{array}{c}\text { Problem } \\
\text { No. }\end{array}$} & \multicolumn{3}{|c|}{ Prev. best by heuristics } & \multicolumn{4}{|c|}{ EVRPTW-CNCF $(\mathrm{L}=2000)$} & \multicolumn{3}{|c|}{ EVRPTW-CNCF $(\mathrm{L}=150)$} \\
\hline & Dis. & Veh. & Author & Dis. & Veh. & Dev.\% & Gap $\%$ & Dis. & Veh. & Gap $\%$ \\
\hline $\mathrm{C} 101$ & 828.94 & 10 & RT & 828.94 & 10 & 0.0 & 29.5 & -- & -- & -- \\
\hline $\mathrm{C} 102$ & 828.94 & 10 & RT & 828.94 & 10 & 0.0 & 71.3 & -- & -- & -- \\
\hline $\mathrm{C} 103$ & 828.94 & 10 & RT & 828.94 & 10 & 0.0 & 86.7 & -- & -- & -- \\
\hline $\mathrm{C} 104$ & 828.94 & 10 & RT & 828.94 & 10 & 0.0 & 94.8 & -- & -- & -- \\
\hline $\mathrm{C} 105$ & 828.94 & 10 & RT & 828.94 & 10 & 0.0 & 68.0 & 1196.30 & 13 & 81.4 \\
\hline C106 & 828.94 & 10 & RT & 828.94 & 10 & 0.0 & 81.0 & 1129.08 & 13 & 86.1 \\
\hline C107 & 828.94 & 10 & RT & 828.94 & 10 & 0.0 & 93.9 & -- & -- & -- \\
\hline C108 & 828.94 & 10 & RT & 828.94 & 10 & 0.0 & 100.0 & 1097.36 & 12 & 100.0 \\
\hline C109 & 828.94 & 10 & RT & 828.94 & 10 & 0.0 & 100.0 & 1097.36 & 12 & 100.0 \\
\hline R101 & 1645.79 & 19 & $\mathrm{H}$ & 1656.51 & 20 & 0.65 & 25.6 & 1642.88 & 20 & 25.8 \\
\hline R102 & 1486.12 & 17 & RT & 1649.26 & 20 & 11.0 & 82.7 & 1595.96 & 19 & 79.1 \\
\hline R103 & 1292.68 & 13 & LLH & 1649.26 & 20 & 27.6 & 96.3 & 1586.95 & 18 & 95.7 \\
\hline R104 & 1007.24 & 9 & M & 1649.26 & 20 & 63.7 & 98.7 & 1566.73 & 18 & 98.0 \\
\hline R105 & 1377.11 & 14 & RT & 1624.14 & 19 & 17.9 & 71.1 & 1423.55 & 16 & 58.8 \\
\hline
\end{tabular}




\begin{tabular}{ccccccccccc} 
R106 & 1251.98 & 12 & M & 1624.14 & 19 & 29.7 & 99.1 & 1420.74 & 16 & 98.0 \\
R107 & 1104.66 & 10 & S97 & 1624.14 & 19 & 47.0 & 99.6 & 1362.22 & 14 & 99.7 \\
R108 & 960.88 & 9 & BBB & 1624.14 & 19 & 69.0 & 99.5 & 1362.22 & 14 & 99.5 \\
R109 & 1194.73 & 11 & HG & 1612.25 & 17 & 34.9 & 100.0 & -- & -- & -- \\
R110 & 1118.59 & 10 & M & - & - & - & - & 2976.20 & 29 & 100.0 \\
R111 & 1096.72 & 10 & RGP & 3200.92 & 25 & 191.8 & 99.6 & 3498.90 & 29 & 99.7 \\
R112 & 982.14 & 9 & GTA & - & - & - & - & 3498.90 & 29 & -- \\
\hline RC101 & 1496.94 & 14 & TBGGP & 1758.69 & 17 & 17.5 & 47.6 & 1690.80 & 17 & 43.7 \\
RC102 & 1554.75 & 12 & TBGGP & 1758.69 & 17 & 13.1 & 98.9 & 1687.69 & 17 & 96.5 \\
RC103 & 1261.67 & 11 & S98 & 1758.69 & 17 & 39.4 & 99.6 & 1674.88 & 16 & 99.3 \\
RC104 & 1135.48 & 10 & CLM & 1758.69 & 17 & 54.9 & 100.0 & 1640.24 & 15 & 100.0 \\
RC105 & 1629.44 & 13 & BBB & 2589.51 & 23 & 58.9 & 89.3 & -- & -- & -- \\
RC106 & 1424.73 & 11 & BBB & 3336.57 & 28 & 134.2 & 99.5 & -- & -- & -- \\
RC107 & 1230.48 & 11 & S97 & - & - & - & - & -- & -- & -- \\
RC108 & 1139.82 & 10 & TBGGP & - & - & - & - & -- & -- & -- \\
\hline
\end{tabular}

\section{Conclusion}

In this study, the EVRPTW is modeled using an efficient linearization approach on the convex nonlinear charging function (CNFC) and a novel approach on routing formulation with unlimited CS visits of EVs and without using dummy CS copies. In comparison to traditional VRPs, the CNFC linearization can be as accurate as needed by adding only one additional variable, and the routing formulation reduces the formulation complexity of the EVRP to the same level as the traditional VRPs, i.e., $\mathrm{O}\left(|N|^{2}\right)$. The proposed MILP model can be solved efficiently with considerations of various practical factors in using EVs, such as CNCF, continuous charging time, safety threshold for the SOC, and comprehensive cost function of using EVs.

Some managerial insights for the practical use of the MILP model were hinted by the computational experiments on Solomon's benchmark instances, as concluded below.

(1) The approximation accuracy of the CNFC linearization was showed to be $1.2 \%$ on average ( $k=8$, type I) in the computational experiments. This slight deviation could be deemed as tolerable in practical applications. Further, the setting for a safety SOC threshold also guaranteed the practical safety of the CNFC linearization.

(2) If the same available time is available to charge an EV in some cases, it will be more efficient to charge EV when its SOC is lower. This rule is efficiently applied in the proposed EVRPTW-CNFC model to take the best utilization of the available charging time.

(3) The model can help the logistics managers to adapt better to the fixed charging time policy for EVs in some resource-intensive scenarios where EVs may not be allowed to occupy a CS by a long time. The optimized CS visits and charging time are driven toward the best utilization of such policy.

The proposed MILP model helps to reduce logistic time with experiments representing the real logistics stituations/scenarios. However, traditional drawbacks of VRPs remain to the VRPTW-CNCF model, such as still NPhard and very difficult to solve for large-sized problems. Future research may focus on developing more efficient heuristic algorithm to solve large-sized problems, and considering the travel speeds time-dependent to make the 
model applicable to time-varying traffic environments.

\section{Acknowledgments}

This study was partially supported by the National Natural Science Foundation of China under Grant Nos. 71871003 and 71271009.

\section{Appendix}

Proposition 1. For a solution, $\pi$, of the MILP model formulated by Eqs. (11)-(22), if the charging time $t_{i}^{\prime \prime}$ in $\pi$ is not zero, then it may have a value that is greater than the actual charging time needed to charge the battery from $p_{i}^{\prime}$ to $p_{i}^{\prime}+p_{i}^{\prime \prime}$.

Proof. Let $t=f(p)$ be the concave nonlinear charge function for an EV and $p=f^{-1}(t)$ is the reverse function, where $t$ is the charging time needed to charge the battery energy from 0 to $p . \pi$ satisfies Constraint (21-5), so the variables $t_{i}^{\prime}$ and $t_{i}^{\prime \prime}$ in $\pi$ satisfy $f^{-1}\left(p_{i}^{\prime}+p_{i}^{\prime \prime}\right) \leq t_{i}^{\prime}+t_{i}^{\prime \prime}$ (see Fig. 1 for the explanation). Thus, the value of $\left(t_{i}^{\prime}+t_{i}^{\prime \prime}\right)$ is allowed to be larger than actually required (to charge the amount of energy $p_{i}^{\prime}+p_{i}^{\prime \prime}$ in the battery). The value of $t_{i}^{\prime}$ is already tightly bounded with respect to $p_{i}^{\prime}$ by Constraints (21-3) and (21-4), so only $t_{i}^{\prime \prime}$ is free and it is possibly larger than that required to chare the battery with energy $p_{i}^{\prime \prime}$. Furthermore, the charging time, $t_{i}^{\prime \prime}$, makes an equally weighted contribution (to the objective function) to the waiting time (i.e., $w_{i}$ and $w_{i}^{\prime}$ ), so $t_{i}^{\prime \prime}$ is allowed to include part of the waiting time without affecting the objective value. Therefore, it is possible for the charging time, $t_{i}^{\prime \prime}$, to be greater than the actual charging time needed to charge the battery from $p_{i}^{\prime}$ to $p_{i}^{\prime}+p_{i}^{\prime \prime}$.

To obtain an accurate value for the charging time, $t_{i}^{\prime \prime}$, the MILP model can be resolved to minimize the total charging time while the total cost of the solution, $\pi$, denoted as $T C(\pi)$, remains unchanged, as follows.

Minimize: Total charging time $=\sum_{i \in N^{\prime \prime}} t_{i}^{\prime \prime}$

Subject to Constraints (12)-(22) and Constraint (24) as follows:

$$
g_{v} \sum_{(0, j) \in A} x_{0 j}+\left(g_{e} C_{e} / L\right) \sum_{(i, j) \in A} D_{i j}+g_{d} \sum_{(i, j) \in A} D_{i j}+g_{t}\left[\sum_{(i, j) \in A} T_{i j}+\sum_{i \in N^{\prime}}\left(w_{i}+z_{i}+w_{i}^{\prime}+t_{i}^{\prime \prime}\right)\right] \leq T C(\pi)
$$

Proposition 2. A feasible solution, $\pi^{*}$, of the MILP model formulated by the objective function in Eq. (23) subject to Constraints (12)-(22) and (24) is also a feasible solution of the MILP model formulated by Eqs. (12)-(22).

Proof. This is straightforward because $\pi^{*}$ satisfies Constraints (12)-(22).

It should be noted that when solving the MILP model with Eq. (23) and Constraints (12)-(22) and (24), the binary decision variables, including $x_{i j}, y_{i c}, y_{i}^{\prime}$, and $\alpha_{i r}$, can be fixed as input parameters to efficiently obtain $\pi^{*}$.

However, even if the charging time, $t_{i}^{\prime \prime}$, is tightly bounded by Constraint (21-5), an approximation error remains between the secant lines and nonlinear curve of the charging function. If the maximum SOC deviation caused by the piecewise linearization is $\varepsilon$, then the following proposition can be applied for judging the actual feasibility of a 
solution.

Proposition 3. The solution obtained by the MILP model formulated by Eq. (23) and Constraints (12)-(22) and (24) is an actual feasible solution of the problem EVRPTW-CNCF if the setting of the SOC safety threshold when arriving at a $\mathrm{CS}$ or depot, i.e., $\lambda$, is greater than or equal to $\varepsilon$.

Proof. Even if the electrical energy charged at a CS is smaller than the expected amount with a maximum deviation of $\varepsilon$, then this difference can be addressed by the setting the SOC safety threshold such that EV is guaranteed to arrive at the next CS or depot. Thus, the solution is actually feasible for the EVRPTW-CNCF problem.

Proposition 4. For a feasible solution, $\pi^{*}$, of the proposed MILP model formulated by Eq. (23) and Constraints (12)(22) and (24), the remaining energy of an EV that has visited a CS is equal to $\lambda$ when returning to the depot.

Proof. This proof is straightforward. The total charging time has been minimized, so the EVs will consistently attempt to spend less time at CSs. Thus, the remaining electrical power will be equal to $\lambda$ when returning to the depot.

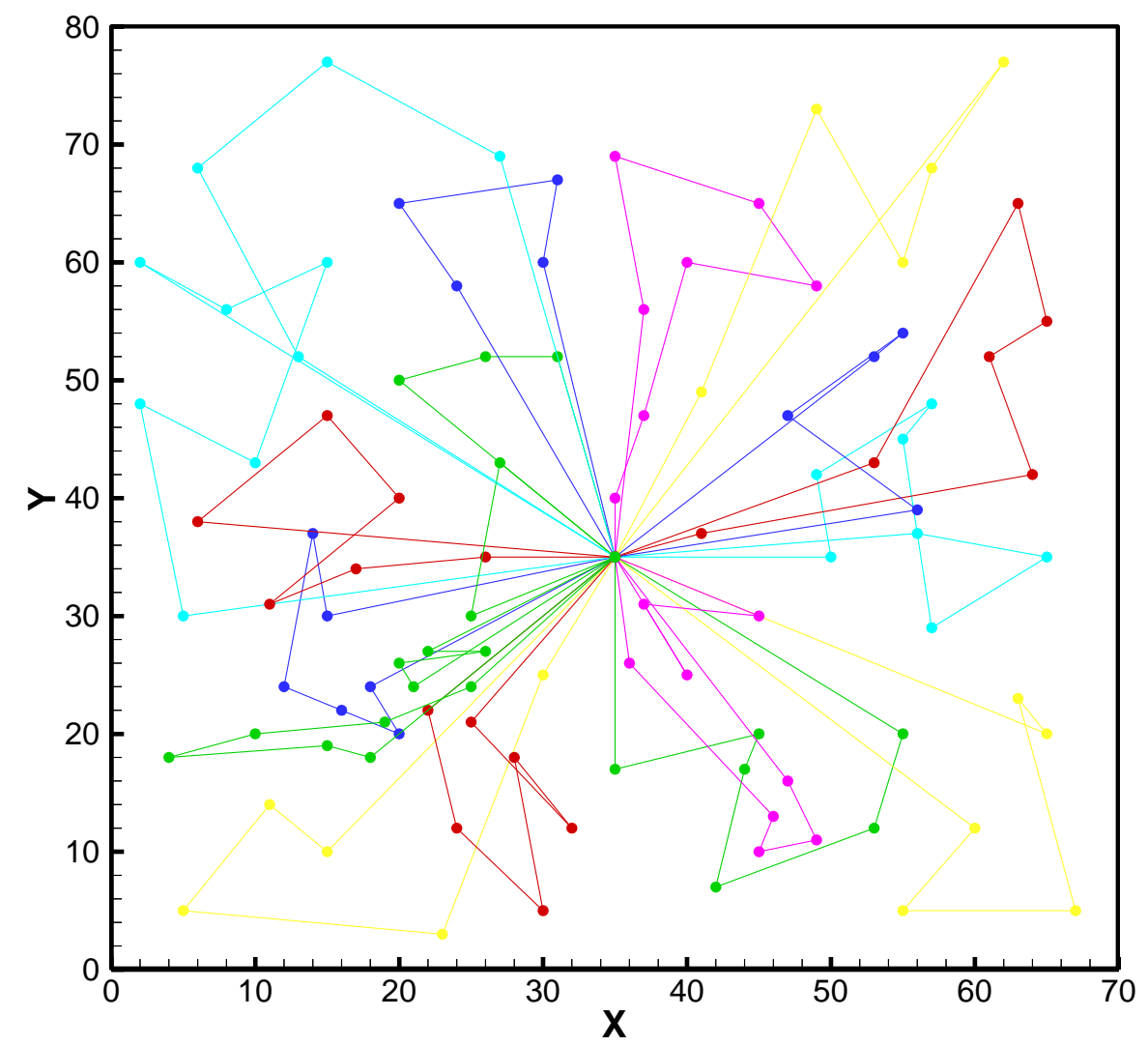

Fig. A1. New best-known solution for R101 (obj.=1642.88)

$(0,2,21,73,41,56,4,0,5,83,61,85,37,93,0,12,76,79,3,54,24,80,0,14,44,38,43,13,0,27,69,30,51,20,32,70,0,28,29,78,34,35,77,0,31,88,7,0$, $33,81,50,68,0,36,47,19,8,46,17,0,39,23,67,55,25,0,40,53,26,0,45,82,18,84,60,89,0,52,6,0,59,99,94,96,0,62,11,90,10,0,63,64,49,48,0,6$ $5,71,9,66,1,0,72,75,22,74,58,0,92,42,15,87,57,97,0,95,98,16,86,91,100,0)$

\section{References}

1. Adler J. D., Mirchandani P. B., 2014. Online routing and battery reservations for electric vehicles with swappable batteries. Transportation Research Part B: Methodological. 70, 285-302.

2. Bahramara S., Golpîra H., 2018. Robust optimization of micro-grids operation problem in the presence of electric vehicles. 
Sustainable cities and society. 37, 388-395.

3. Bektaş T., Laporte G., 2011. The pollution-routing problem. Transportation Research Part B: Methodological. 45(8), 1232-1250.

4. Bruglieri M., Pezzella F., Pisacane O., Suraci S., 2015. A variable neighborhood search branching for the electric vehicle routing problem with time windows. Electronic Notes in Discrete Mathematics. 47, 221-228.

5. Casals L. C., Martinez-Laserna E., García B. A., Nieto N., 2016. Sustainability analysis of the electric vehicle use in Europe for CO2 emissions reduction. Journal of Cleaner Production 127, 425-437.

6. Conrad R. G., Figliozzi M. A., 2011. The recharging vehicle routing problem. IIE Annual Conference. Proceedings. Institute of Industrial and Systems Engineers (IISE). Reno, Nevada. 1.

7. Croxton, K., Gendron, B., and Magnanti, T. (2003). A Comparison of Mixed-Integer Programming Modelsfor nonconvex Piecewise Linear Cost Minimization Problems. Management Science, 49(3):1268-1273.

8. Dantzig G. B., Ramser J. H., 1959. The truck dispatching problem. Management science. 6(1), 80-91.

9. Demir E., Bektaş T., Laporte G., 2012. An adaptive large neighborhood search heuristic for the pollution-routing problem. European Journal of Operational Research. 223(2), 346-359.

10. Desaulniers G., Errico F., Irnich S., Schneider M., 2016. Exact algorithms for electric vehicle-routing problems with time windows. Operations Research. 64(6), 1388-1405.

11. Erdem M., Koç Ç., 2019. Analysis of electric vehicles in home health care routing problem. Journal of Cleaner Production, In press, https://doi.org/10.1016/j.jclepro.2019.06.236.

12. Erdoğan S., Miller-Hooks E., 2012. A green vehicle routing problem. Transportation Research Part E: Logistics and Transportation Review. 48(1), 100-114.

13. Fathollahi-Fard, A.M., Hajiaghaei-Keshteli, M., Mirjalili, S., 2019. A set of efficient heuristics for a home healthcare problem. Neural Comput \& Applic 2019, 1-21.

14. Fathollahi-Fard A. M., Hajiaghaei-Keshteli M., Tavakkoli-Moghaddam R., 2018a. A bi-objective green home health care routing problem. Journal of Cleaner Production 200, 423-443.

15. Fathollahi-Fard A. M., Hajiaghaei-Keshteli M., Tavakkoli-Moghaddam R., 2018b. A Lagrangian relaxation-based algorithm to solve a Home Health Care routing problem. International Journal of Engineering 31(10), 1734-1740.

16. Fathollahi-Fard A. M., Hajiaghaei-Keshteli M., 2018. Integrated Capacitated Transportation and Production Scheduling Problem in a Fuzzy Environment. International Journal of Industrial Engineering \& Production Research 29(2), 197-211.

17. Fernandez R. A., 2018. A more realistic approach to electric vehicle contribution to greenhouse gas emissions in the city. Journal of Cleaner Production 172, 949-959.

18. Felipe Á., Ortuño M. T., Righini G., Tirado G., 2014. A heuristic approach for the green vehicle routing problem with multiple technologies and partial recharges. Transportation Research Part E: Logistics and Transportation Review. 71, 111-128.

19. Figliozzi M., 2010. Vehicle routing problem for emissions minimization. Transportation Research Record: Journal of the Transportation Research Board. 2197, 1-7.

20. Froger A., Mendoza J.E., Jabali O., Laporte G., 2017a. New Formulations for the Electric Vehicle Routing Problem with Nonlinear Charging Functions. Diss. Centre interuniversitaire de recherche sur les reseaux d'entreprise, la logistique et le transport (CIRRELT).

21. Froger A., Mendoza J.E., Jabali O., Laporte G. 2017b. A matheuristic for the Electric Vehicle Routing Problem with Capacitated Charging Stations. Diss. Centre interuniversitaire de recherche sur les reseaux d'entreprise, la logistique et le transport (CIRRELT).

22. Fu Y., Tian G., Fathollahi-Fard A. M., Ahmadi A., Zhang C., 2019. Stochastic multi-objective modelling and optimization of an energy-conscious distributed permutation flow shop scheduling problem with the total tardiness constraint. Journal of Cleaner Production 226(20), 515-525.

23. Gayialis S. P., Konstantakopoulos G. D., Tatsiopoulos I. P., 2019. Vehicle Routing Problem for Urban Freight Transportation: A Review of the Recent Literature. Operational Research in the Digital Era-ICT Challenges. 89-104.

24. Goeke D., Schneider M., 2015. Routing a mixed fleet of electric and conventional vehicles. European Journal of Operational 
Research. 245(1), 81-99.

25. Golden B. L., Raghavan S., Wasil E., 2008. The vehicle routing problem: latest advances and new challenges. Springer Science \& Business Media. 43.

26. Golpîra H., Khan S. A. R., 2019. A multi-objective risk-based robust optimization approach to energy management in smart residential buildings under combined demand and supply uncertainty. Energy. 170, 1113-1129.

27. Hiermann G., Puchinger J., Ropke S., Hartl R. F., 2016. The electric fleet size and mix vehicle routing problem with time windows and recharging stations. European Journal of Operational Research 252(3), 995-1018.

28. Hof J., Schneider M., Goeke D., 2017. Solving the battery swap station location routing problem with capacitated electric vehicles using an AVNS algorithm for vehicle-routing problems with intermediate stops. Transportation Research Part B: Methodological 97, 102-112.

29. International Energy Agency (IEA), 2018. Global EV outlook 2018.

30. Jabali O., Woensel T., De Kok A. G., 2012. Analysis of travel times and CO2 emissions in time-dependent vehicle routing. Production and Operations Management. 21(6), 1060-1074.

31. Jie W., Yang J., Zhang M., Huang Y., 2018. The two-echelon capacitated electric vehicle routing problem with battery swapping stations: formulation and efficient methodology. European Journal of Operational Research 272 (3), 879-904.

32. Kara I., Kara B. Y., Yetis M. K., 2007. Energy minimizing vehicle routing problem. International Conference on Combinatorial Optimization and Applications. 62-71.

33. Keskin M., Çatay B., 2016. Partial recharge strategies for the electric vehicle routing problem with time windows. Transportation Research Part C: Emerging Technologies. 65, 111-127.

34. Koç Ç., Karaoglan I., 2016. The green vehicle routing problem: A heuristic based exact solution approach. Applied Soft Computing. $39,154-164$.

35. Laporte G., Gendreau M., Potvin J. Y., Semet F., 2000. Classical and modern heuristics for the vehicle routing problem. International Transactions in Operational Research. 7(4-5), 285-300.

36. Leggieri V., Haouari M., 2017. A practical solution approach for the green vehicle routing problem. Transportation Research Part E: Logistics and Transportation Review. 104, 97-112.

37. Letchford A. N., Salazar-González J. J., 2019. The capacitated vehicle routing problem: stronger bounds in pseudo-polynomial time. European Journal of Operational Research. 272(1), 24-31.

38. Liao C. S., Lu S. H., Shen Z. J. M., 2016. The electric vehicle touring problem. Transportation Research Part B: Methodological. 86, 163-180.

39. Lin C., Choy K., Ho G., Chung S., Lam H., 2014. Survey of green vehicle routing problem: Past and future trends. Expert Systems with Applications. 41(4), 1118-1138.

40. Lin J., Zhou W., Wolfson O., 2016. Electric vehicle routing problem. Transportation Research Procedia. 12, 508-521.

41. Ma S.-C., Fan Y., Guo J.-F., Xu J.-H., Zhu J., 2019. Analysing online behaviour to determine Chinese consumers' preferences for electric vehicles. Journal of Cleaner Production 229, 244-255.

42. Madankumar S., Rajendran C., 2019. A mixed integer linear programming model for the vehicle routing problem with simultaneous delivery and pickup by heterogeneous vehicles, and constrained by time windows. Sādhanā. 44(2), 39.

43. Montoya A., Guéret C., Mendoza J. E., Villegas J. G., 2017. The electric vehicle routing problem with nonlinear charging function. Transportation Research Part B: Methodological. 103, 87-110.

44. Sahin B., Yilmaz H., Ust Y., Guneri A. F., Gulsun B., 2009. An approach for analysing transportation costs and a case study. European Journal of Operational Research. 193(1), 1-11.

45. Schiffer M., Walther G., 2017. The electric location routing problem with time windows and partial recharging. European Journal of Operational Research. 260(3), 995-1013.

46. Schneider M., Stenger A., Goeke D., 2014. The electric vehicle-routing problem with time windows and recharging stations. Transportation Science. 48(4), 500-520. 
47. Soleimani H., Chaharlang Y., Ghaderi H., 2018. Collection and distribution of returned-remanufactured products in a vehicle routing problem with pickup and delivery considering sustainable and green criteria.Journal of Cleaner Production 172, 960-970.

48. Strehler M., Merting S., Schwan C., 2017. Energy-efficient shortest routes for electric and hybrid vehicles. Transportation Research Part B: Methodological. 103, 111-135.

49. Sweda T. M., Dolinskaya I. S., Klabjan D., 2016. Optimal recharging policies for electric vehicles. Transportation Science. 51(2), 457-479.

50. TeslaFan T., 2015. Model S supercharging times compared S60, S70D, S85, P85D, S85D. https://forums.tesla.com/forum/forums/model-s-supercharging-times-compared-s60-s70d-s85-p85d-s85d [accessed 5/19/2019].

51. Toth P., Vigo D., 2002. The vehicle routing problem. Society for Industrial and Applied Mathematics. 9, 1-26.

52. Villegas J., Guéret C., Mendoza J. E., Montoya A., 2018. The technician routing and scheduling problem with conventional and electric vehicle. Diss. Centre interuniversitaire de recherche sur les reseaux d'entreprise, la logistique et le transport (CIRRELT).

53. Wang Y., Zhang S., Assogba K., Fan J., Xu M., Wang Y., 2018. Economic and environmental evaluations in the two-echelon collaborative multiple centers vehicle routing optimization. Journal of Cleaner Production 197(1), 443-461.

54. Wu Z., Wang M., Zheng J., Sun X., Zhao M., Wang X., 2018. Life cycle greenhouse gas emission reduction potential of battery electric vehicle. Journal of Cleaner Production 190, 462-470.

55. Xiao Y., Konak A., 2015. A simulating annealing algorithm to solve the green vehicle routing \& scheduling problem with hierarchical objectives and weighted tardiness. Applied Soft Computing. 34, 372-388.

56. Xiao Y., Konak A., 2016. The heterogeneous green vehicle routing and scheduling problem with time-varying traffic congestion. Transportation Research Part E: Logistics and Transportation Review. 88, 146-166.

57. Xiao Y., Konak A., 2017. A genetic algorithm with exact dynamic programming for the green vehicle routing \& scheduling problem. Journal of Cleaner Production. 167, 1450-1463.

58. Xiao Y., Zhao Q., Kaku I., Xu Y., 2012. Development of a fuel consumption optimization model for the capacitated vehicle routing problem. Computers \& Operations Research. 39(7), 1419-1431.

59. Xiao Y., Zuo X., Kaku I., Zhou S., Pan X., 2019. Development of energy consumption optimization model for the electric vehicle routing problem with time windows. Journal of Cleaner Production. 225, 647-663.

60. Yang J., Sun H., 2015. Battery swap station location-routing problem with capacitated electric vehicles. Computers \& Operations Research. 55, 217-232.

61. Yu A. S. O., Silva L. L. C., Chu C. L., Nascimento P. T. S., Camargo A. S., 2011. Electric vehicles: Struggles in creating a market. Technology Management in the Energy Smart World (PICMET). 1-13.

62. Zhang J., Zhao Y., Xue W., Li J., 2015. Vehicle routing problem with fuel consumption and carbon emission. International Journal of Production Economics. 170, 234-242.

63. Zhang Q., Ou X., Yan X., Zhang X., 2017. Electric vehicle market penetration and impacts on energy consumption and CO2 emission in the future: Beijing case. Energies. 10(2), 228.

64. Zheng Y., Dong Z. Y., Xu Y., Meng K., Zhao J. H., Qiu J., 2014. Electric vehicle battery charging/swap stations in distribution systems: comparison study and optimal planning. IEEE Transactions on Power Systems. 29(1), 221-229.

65. Zuo X., Zhu C., Huang C., Xiao Y., 2017. Using AMPL/CPLEX to model and solve the electric vehicle routing problem (EVRP) with heterogeneous mixed fleet. Control and Decision Conference (CCDC). 4666-4670. 